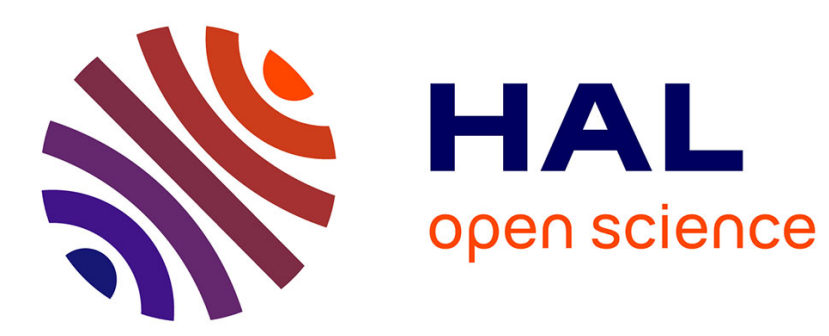

\title{
Genericity of the strong observability for sampled
}

Sabeur Ammar, Jean-Claude Vivalda, Majid Massaoud

\section{To cite this version:}

Sabeur Ammar, Jean-Claude Vivalda, Majid Massaoud. Genericity of the strong observability for sampled. SIAM Journal on Control and Optimization, 2018, 56 (2), pp.28. 10.1137/16M1084961. hal-01630461

\section{HAL Id: hal-01630461 \\ https://hal.inria.fr/hal-01630461}

Submitted on 7 Nov 2017

HAL is a multi-disciplinary open access archive for the deposit and dissemination of scientific research documents, whether they are published or not. The documents may come from teaching and research institutions in France or abroad, or from public or private research centers.
L'archive ouverte pluridisciplinaire HAL, est destinée au dépôt et à la diffusion de documents scientifiques de niveau recherche, publiés ou non, émanant des établissements d'enseignement et de recherche français ou étrangers, des laboratoires publics ou privés. 


\title{
GENERICITY OF THE STRONG OBSERVABILITY FOR SAMPLED SYSTEMS.
}

\author{
SABEUR AMMAR*, MAJID MASSAOUD ${ }^{\dagger}$, AND JEAN-CLAUDE VIVALDA $\ddagger$
}

Abstract. In this paper we prove that, generically, a sampled data system is observable provided that the number of outputs is greater than the number of inputs plus 1 .

Key words. Observability, Sampled systems, Genericity,Transversality

AMS subject classifications. 93B07, 93C57

1. Introduction. In this paper we deal with the genericity of the observability of sampled data systems. Consider a controlled continuous time system written as

$$
\left\{\begin{array}{l}
\dot{x}=f(x, u) \\
y=h(x)
\end{array}\right.
$$

Given a time $T$, to system (1), we relate the following continuous-discrete-time system

$$
\left\{\begin{aligned}
\dot{x}(t) & =f\left(x(t), u_{k}\right), t \in[k T,(k+1) T) \\
y_{k} & =h(x(k T))
\end{aligned}\right.
$$

where the control $u$ is maintained constant on the intervals $[k T,(k+1) T)$ and the measurements of the state are made only at each of the times $0, T, 2 T, \ldots$ System (2) is called the sampled data system related to (1).

Many physical processes or industrial devices can be modeled by a system of continuous-time differential equations as (1). From a mathematical viewpoint, the time and the state of this system can vary continuously but in practice, a controlled process is regulated by a digital computer which is not able to record a continuum of data. This is why control decisions are restricted to be taken at fixed times $0, T, 2 T, \ldots$; here $T$ is called the sampling time and is a (generally small) parameter which depends on the instrumentation of the process, on the computing power and other parameters. For a continuous time system, the resulting situation can be modeled through the restriction that the applied inputs are constant on the intervals $[0, T),[T, 2 T), \ldots$ and the state is (partially) measured only at those fixed times $0, T, 2 T, \ldots$, that is to say we access to the values of the observation function only at times $0, T, \ldots$

For the sake of clarity, the precise assumptions that we make on these systems are stated in section 1.1 but we recall here the notion of observability. Regarding system (2), an input $u^{0}$ is a sequence $\left(u_{k}\right)_{k \geq 0}$ with $u_{k} \in U$ (U, the input space). An input $u^{0}$ being given, we denote by $x(t)$ and $\bar{x}(t)$ the solutions of (2) starting from $x_{0}$ and $\bar{x}_{0}$ respectively; we say that system (2) is observable for $u^{0}$ if for every pair of initial conditions $\left(x_{0}, \bar{x}_{0}\right)$, there exists an integer $k$ such that $h(x(k T)) \neq h(\bar{x}(k T))$.

${ }^{*}$ S. Ammar is with Institut Supérieur d'Informatique et du Multimédia de Sfax, Université de Sfax, ammar_3021@yahoo.fr

† M. Massaoud is with FSS Sfax, Université de Sfax, Tunisia, majidmassaoud@gmail.com

$\ddagger$ J.-C. Vivalda is with Inria, Villers-lès-Nancy, F-54600, France- Université de Lorraine, IECL, UMR 7502, Vandœuvre-lès-Nancy, F-54506, France — CNRS, IECL,UMR 7502, Vandœuvre-lèsNancy, F-54506, France Jean-Claude.Vivalda@inria.fr 
The aim of this paper is to prove that, under some conditions on the respective dimensions of the inputs and the output, generically, the sampled system obtained from a continuous time system is observable.

Two questions can be investigated about observability and sampled systems. The first one is the problem of the preservation of the observability: if the continuous time system (1) is observable for any inputs, is it also the case for the sampled system (2)? The second question is the subject of this paper: given a sampling time $T$, how many are the continuous time systems (1) such that the sampled system (2) is observable?

Concerning the first question, the answer for linear systems is well known (see e.g. [18] and also [14] when the sampling time is not constant). If we deal with nonlinear systems, one would think that the observability of the continuous time system involves the observability of the sampled one, at least if the sampling time is chosen small enough. Surprisingly, this is not the case: a counter-example can be found in [6]; in order to get the observability of the sampled system, to this natural condition (the observability of the continuous time system), we have to add the condition of infinitesimal observability (see [11]) together with a technical condition bearing on the sequence of controls $u_{0}, u_{1}, \ldots$

The aim of this paper lies on a more "philosophical plane". Due to the importance of the notion of observability, it is of interest to know "how many" continuous time systems give rise to observable sampled data systems. To be more precise, in [6] (systems given on a compact manifold) and in [3,5] (systems given on $\mathbf{R}^{n}$ ), we gave some natural sufficient conditions bearing on the continuous time system and under which the sampled system is observable. So, these works have a practical interest: for a class of continuous time systems our result allows us to decide on the observability of the sampled system. The present paper intends to prove that the set of continuous time systems which admit an observable sampled system is everywhere dense. Knowing that the set of rational numbers is dense in the set of real numbers does not permit us to decide if a particular given number is rational; in the same way, the result proven in this paper does not permit to decide if a particular sampled system is observable. Moreover, this result cannot be deduced from the abovementioned papers because, while the observability of the continuous time system is generic (see [11]) the additional conditions in $[6,3,5]$ are not; also, in these papers, the observability is ensured only for sufficiently small sampling time $T$.

The genericity of the observability has been the subject of some researches in the last decades. As regards continuous-time systems, the first paper on the subject was about the genericity of the observability for uncontrolled systems [9]; this work was generalized to controlled systems by J.-P. Gauthier and I. Kupka, in [10] these authors proved the genericity of differential observability for systems with more outputs than inputs. A reference book on this subject is [11]. A related issue is the problem of the identifiability, in [8], the authors deal with general nonlinear systems which contain an unknown function, they prove that these (uncontrolled) systems are generically identifiable if the number of observations is at least three. Regarding the discretetime systems, the first paper on the subject was from Aeyels [2], we can cite also [20] for the uncontrolled case and [7,4] for the controlled case. In all of these papers, it is proved that the observability is a generic property provided that the number of outputs is greater than the number of inputs. Surprisingly, this result is no more valid for the systems considered in this paper: in the next sections, we shall prove that if the number of inputs is one and the number of outputs is two, the set of pairs $(f, h)$ such that system (2) is observable is not dense. Concerning the subject of this paper, we have also to cite [13], in this paper the authors prove also a result of genericity of 
the observability for sampled data systems; the systems considered in this paper are uncontrolled and the sampling time is not constant but depends on the sate of the system.

The tools used to prove our main result are essentially the same (but applied to different situations) as the ones used in the above-mentioned papers, that is to say the major theorem of the transversality theory.

The paper is organized as follows: in the next section, we state the precise formulation of the problem we deal with and we recall some useful facts from the transversality theory. In section 2, we state the main result of the paper and we introduce the lists $L_{2 n}$ and $\bar{L}_{2 n}$, which are built from two initial conditions $x_{0}$ and $\bar{x}_{0}$ and their images under the iterates of $f$; we then introduce five possible configurations fore these lists ( $c f$ section 2.1). We then prove our main theorem for each of these configurations: the corresponding results are stated in the three propositions 5, 9 and 10; the conjunction of these propositions give the main result. Finally, we give a counter-example in order to prove that the observability of the sampled system is not generic in the case $d_{u}=d_{y}+1$ (cf. section 5).

1.1. Problem formulation. We consider two compact manifolds $X$ and $U$; we let $n=\operatorname{dim} X$ and $d_{u}=\operatorname{dim} U$. As usual we denote by $\mathrm{T}_{x} X$ the tangent space to $X$ at $x$, and by $\mathrm{T} X$ the tangent bundle. A parametrized vector field will be a $C^{\infty}$ mapping defined from $X \times U$ into $\mathrm{T} X$ such that, for every $u \in U, f(\cdot, u)$ is a vector field defined on $X$. The set of parametrized vector fields defined on $X$ will be denoted by $\Gamma_{U}(X)$. If $f$ belongs to $\Gamma_{U}(X)$, we denote by $\varphi_{t}^{u}$ the flow generated by the vector field $f(\cdot, u)$ (the parameter $u$ being fixed); so for every $x \in X$, every $u \in U$, and every $t \geq 0$, we have

$$
\varphi_{0}^{u}(x)=x \quad \text { and } \quad \frac{\mathrm{d} \varphi_{t}^{u}(x)}{\mathrm{d} t}=f\left(\varphi_{t}^{u}(x), u\right) .
$$

Let $u_{0}, u_{1}, \ldots$ be a sequence of controls (i.e. a sequence of elements of $U$ ), for $k \geq 1$, we denote by $u_{k}$ the finite sequence $u_{k}=\left(u_{0}, \ldots, u_{k-1}\right)$.

Let $\psi: M \rightarrow N$ be a differentiable mapping between two manifolds $M$ and $N$, the notation $\mathrm{d} \psi(x)$ will stand for the differential of $\psi$ at $x$; let $\xi \in \mathrm{T}_{x} M$ be a tangent vector, $\mathrm{d} \psi(x) \cdot \xi$ will denote the image of $\xi$ under $\mathrm{d} \psi(x)$.

Hereafter, together with a parametrized vector field, we consider a $C^{\infty}$ mapping $h$ from $X$ to $\mathbf{R}^{d_{y}}$ and, given a sampling time $T>0$, we consider the mapping $\Theta_{T}^{f, h}$ defined as

$$
\begin{aligned}
\Theta_{T}^{f, h}: X \times U^{2 n} & \longrightarrow \mathbf{R}^{(2 n+1) d_{y} \times U^{2 n}} \\
\left(x, \underline{u_{2 n}}\right) & \longmapsto\left(h\left(x_{0}\right), h\left(x_{1}\right), \ldots, h\left(x_{2 n}\right), \underline{u_{2 n}}\right)
\end{aligned}
$$

where the sequence $\left(x_{0}, x_{1}, \ldots, x_{2 n}\right)$ is defined recursively by $x_{0}=x$ and $x_{k+1}=$ $\varphi_{T}^{u_{k}}\left(x_{k}\right)$. Also, we denote by $y_{i}$ and $\bar{y}_{i}$, the values at $x_{i}$ and $\bar{x}_{i}$ under $h: y_{i}=h\left(x_{i}\right)$ and $\bar{y}_{i}=h\left(\bar{x}_{i}\right)$.

Definition 1. We shall say that the sampled data system (2) is strongly observable if the mapping $\Theta_{T}^{f, h}$ defined above is one-to-one.

We shall show that, generically, system (2) is strongly observable, to be more precise, we endow $\Gamma_{U}(X) \times C^{\infty}\left(X, \mathbf{R}^{d_{y}}\right)$ with the Whitney topology and we shall prove that the set of pairs $(f, h)$ such that the mapping $\Theta_{T}^{f, h}$ is injective is a residual subset of $\Gamma_{U}(X) \times C^{\infty}\left(X, \mathbf{R}^{d_{y}}\right)$ provided that $d_{y} \geq d_{u}+2$ (case $\left.d_{u}>0\right)$ or $d_{y} \geq 1$ (case $d_{u}=0$ ). The tools used in this paper come from the transversality theory, hereafter, we recall the notion of transversality as well as the Abraham's theorem of density [1] which will be intensively in the proof of our main result. 
DEFINITION 2 (Transversality). Let $f$ be a smooth mapping between two smooth manifolds $X$ and $Y, W$ a submanifold of $Y$ and $x$ a point in $X$. We shall say that $f$ is transversal to $W$ at $x$ if either

- $f(x) \notin W$, or

- $f(x) \in W$ and $\mathrm{T}_{f(x)} Y=\mathrm{T}_{f(x)} W+\mathrm{d} f_{x}\left(\mathrm{~T}_{x} X\right)$.

We shall say that $f$ is transversal to $W$ if it is transversal to $W$ at every $x \in X$. We shall use the symbol $\pitchfork$ to denote the transversality.

Concerning this definition, some elementary conditions show that the second equality cannot be satisfied if $\operatorname{codim} W>\operatorname{dim} X$. Therefore if $\operatorname{codim} W>\operatorname{dim} X$, transversality means non membership: in this case saying that $f$ is transverse to $W$ amounts to saying that $f(x) \notin W$ for every $x \in X$. This trick will be used later in the proofs of propositions 9 and 10 .

We recall also the notion of representation: let $\mathscr{A}, X$ and $Y$ be $C^{r}$ manifolds and $\rho$ a map from $\mathscr{A}$ to $C^{r}(X, Y)$. For $a \in \mathscr{A}, \rho_{a}: X \rightarrow Y$ is the map defined as $\rho_{a}(x)=\rho(a)(x)$. We say that $\rho$ is a $C^{r}$ representation if the evaluation map:

$$
\begin{aligned}
& \mathrm{ev}_{\rho}: \mathscr{A} \times X \quad \longrightarrow Y \\
& (a, x) \quad \longmapsto \quad \rho_{a}(x)=\rho(a)(x)
\end{aligned}
$$

is a $C^{r}$ map from $\mathscr{A} \times X$ to $Y$.

Theorem 3 (Transversal density theorem). Let $\mathscr{A}, X, Y$ be $C^{r}$ manifolds, $\rho$ : $\mathscr{A} \rightarrow C^{r}(X, Y)$ a $C^{r}$ representation, $W \subset Y$ a submanifold (not necessarily closed), and $\mathrm{ev}_{\rho}: \mathscr{A} \times X \rightarrow Y$ the evaluation map. Define $\mathscr{A}_{W} \subset \mathscr{A}$ by:

$$
\mathscr{A}_{W}=\left\{a \in \mathscr{A} \mid \rho_{a} \pitchfork W\right\}
$$

Assume that:

1. $X$ has a finite dimension $n$ and $W$ has a finite codimension $q$ in $Y$;

2. $\mathscr{A}$ and $X$ are second countable;

3. $r>\max (0, n-q)$;

4. $\operatorname{ev}_{\rho} \pitchfork W$.

Then $\mathscr{A}_{W}$ is residual in $\mathscr{A}$.

Notice that manifold $\mathscr{A}$ is not necessarily finite dimensional; it may be a Banach space or an open subset of a Banach space.

\section{Main result.}

THeOREM 4. Assume that $d_{y} \geq d_{u}+2$ or that $d_{u}=0$ and $d_{y} \geq 1$, and let $T>0$ a given sampling time. Then the set of pairs $(f, h)$ such that system (2) is strongly observable is a residual subset of $\Gamma_{U}(X) \times C^{\infty}\left(X, \mathbf{R}^{d_{y}}\right)$.

In order to prove this theorem, we need some preliminary results, namely the propositions 5, 9 and 10 stated in sections 3 and 4.3-4.4. In these propositions, different possible configurations, denoted hereafter by $\mathbf{C}_{\mathbf{0}}$ through $\mathbf{C}_{\mathbf{4}}$, of the lists $\left(x_{0}, x_{1}, \ldots, x_{2 n}\right)$ and $\left(\bar{x}_{0}, \bar{x}_{1}, \ldots, \bar{x}_{2 n}\right)$ are considered (the $\bar{x}_{i}^{\prime} s$ are defined as the $x_{i}$ 's, cf. (3)). For each of this configuration $\mathbf{C}_{\mathbf{k}}(k=0, \ldots, 4)$, we prove that, generically $\Theta_{T}^{f, h}\left(x, \underline{u_{2 n}}\right) \neq \Theta_{T}^{f, h}\left(\bar{x}, \underline{u_{2 n}}\right)$ if $\left(x, \underline{u_{2 n}}\right),\left(\bar{x}, \underline{u_{2 n}}\right)$ is under $\mathbf{C}_{\mathbf{k}}$ configuration.

To be more precise, given two different initial conditions $x$ and $\bar{x}$ and an integer $s \leq 2 n$, we shall consider the two lists

$$
L_{s}=\left(x_{0}, \ldots, x_{s}\right)
$$

$$
\bar{L}_{s}=\left(\bar{x}_{0}, \ldots, \bar{x}_{s}\right),
$$


where the $\bar{x}_{i}$ 's are defined as the $x_{i}$ 's. Concerning the two lists $L_{2 n}$ and $\bar{L}_{2 n}$, we shall examine all the possible situations: the elements of these lists can be pairwise distinct, some equalities can occur among the elements of the first list while the ones of the second are pairwise distinct, etc. In the following section, we define five possible configurations and we prove that, necessarily the above-mentioned lists belong to one of five configurations.

2.1. The different configurations. Hereafter we shall give an exhaustive list of all the possibilities concerning the equalities between the elements of the lists $L_{2 n}$ and $\bar{L}_{2 n}$; in the sequel, we shall say that the equalities $x_{i}=\bar{x}_{j}$ and $x_{i^{\prime}}=\bar{x}_{j^{\prime}}$ between elements of $L_{2 n}$ and elements of $\bar{L}_{2 n}$ are in the same direction if the differences $i-j$ and $i^{\prime}-j^{\prime}$ have the same sign. Take $x \neq \bar{x} \in X$, even if we have to invert the roles of $L_{2 n}$ and $\bar{L}_{2 n}$, the possible configurations for these lists are:

$\mathbf{C}_{\mathbf{0}}$ The elements of $\bar{L}_{2 n}$ are pairwise different; moreover the only possible equalities between elements of $L_{2 n}$ and elements of $\bar{L}_{2 n}$ are all in the "same direction". That is to say, let

$$
I=\left\{0 \leq i \leq 2 n \mid \exists j \in\{0, \ldots, 2 n\}, x_{i}=\bar{x}_{j}\right\},
$$

for $i \in I$, let $E_{i}=\left\{0 \leq j \leq 2 n \mid x_{i}=\bar{x}_{j}\right\}$, then either for every $i \in I$, for every $j \in E_{i}, j<i$ or for every $i \in I$, for every $j \in E_{i}, j>i$.

$\mathbf{C}_{\mathbf{1}}$ There exist some subscripts $0 \leq i<p \leq 2 n$ and $0 \leq j, m \leq 2 n$ such that

- $x_{p}=x_{i}$ and $\bar{x}_{m}=x_{j}$;

- there is no equality between the elements of $L_{p-1}$;

- letting $q=\max (j, p, m)$, there is no equality between the elements of $\bar{L}_{q-1}$;

- the equalities between elements of $L_{q-1}$ and $\bar{L}_{q-1}$ have the same direction.

$\mathbf{C}_{2}$ There exist some subscripts $0 \leq i<p \leq 2 n$, and $0 \leq j<m \leq 2 n$, with $m \geq p$ and such that

- $x_{p}=\bar{x}_{m}$ and $x_{i}=\bar{x}_{j}$ with $p-i \neq m-j$;

- there is no equalities between the elements of $L_{p}$ nor between the elements of $\bar{L}_{m}$;

- the only possible equalities between elements of $L_{m-1}$ and $\bar{L}_{m-1}$ are all in the same direction; moreover if these equalities write $x_{i_{1}}=\bar{x}_{j_{1}}, \ldots$, $x_{i_{r}}=\bar{x}_{j_{r}}$ the differences $i_{1}-j_{1}, \ldots, i_{r}-j_{r}$ are equal;

- if $x_{i^{\prime}}=\bar{x}_{j^{\prime}}$ with $i^{\prime}, j^{\prime} \leq m$, then $i^{\prime} \geq i$ and $j^{\prime} \geq j$.

$\mathbf{C}_{3}$ There exist some subscripts $0 \leq i<p \leq 2 n$, and $0 \leq m<j \leq 2 n$ with $p \leq j$ and such that

- $x_{p}=\bar{x}_{m}$ and $x_{i}=\bar{x}_{j}$

- there is no equalities between the elements of $L_{p}$ nor between the elements of $\bar{L}_{j}$;

- the only possible equalities between elements of $L_{j-1}$ and $\bar{L}_{j-1}$ are all in the same direction; moreover, if these equalities write $x_{i_{1}}=$ $\bar{x}_{j_{1}}, \ldots, x_{i_{r}}=\bar{x}_{j_{r}}$ the differences $i_{1}-j_{1}, \ldots, i_{r}-j_{r}$ are equal;

- if $x_{i^{\prime}}=\bar{x}_{j^{\prime}}$ with $i^{\prime}, j^{\prime} \leq j$, then $i^{\prime} \geq p$ and $j^{\prime} \geq m$.

$\mathbf{C}_{4}$ There exist some subscripts $0 \leq i<p \leq 2 n$, and $0 \leq j<m \leq 2 n$ with $m \geq p$ and such that

- $x_{p}=x_{i}$ and $\bar{x}_{m}=\bar{x}_{j}$;

- there is no equality between one element of $L_{m-1}$ and one element of $\bar{L}_{m-1}$. 
Denote by $e_{s}$ the number of equalities between the elements of $L_{s} \cup \bar{L}_{s}$, if $e_{2 n}=0$ or 1 , then we are under the $\mathbf{C}_{\mathbf{0}}$ configuration.

Now, assume that $e_{2 n} \geq 2$; if we go from $L_{s} \cup \bar{L}_{s}$ to $L_{s-1} \cup \bar{L}_{s-1}$ we lose 0,1 or 2 equalities. Thus if $e_{2 n} \geq 2$, there exists a subscript $m \leq 2 n$ such that there exist exactly two or exactly three equalities between the elements of $L_{m} \cup \bar{L}_{m}$; we denote by $s$ the minimal subscript with this property. Notice that, if $e_{s}$ is exactly three, $x_{s}$ and $\bar{x}_{s}$ must be equal to some elements of $L_{s-1} \cup \bar{L}_{s-1}$ (because we always have $x_{s} \neq \bar{x}_{s}$ ).

Assume first that $e_{s}=3$ and denote by $x_{s}=z_{1}, \bar{x}_{s}=z_{2}$ and $z_{3}=z_{4}$ the three equalities, then

- if $z_{1} \in L_{s}$, and $z_{3}, z_{4} \in L_{s}$, then $L_{s-1}$ and $\bar{L}_{s}$ are under $\mathbf{C}_{\mathbf{1}}$ (if $z_{2} \in L_{s}$ ) or $\mathbf{C}_{4}$ configuration (if $z_{2} \in \bar{L}_{s}$ );

- if $z_{1} \in L_{s}, z_{3} \in L_{s}$, and $z_{4} \in \bar{L}_{s}$, then $L_{s}$ and $\bar{L}_{s-1}$ are under $\mathbf{C}_{\mathbf{1}}$ configuration;

- if $z_{1} \in L_{s}, z_{3} \in \bar{L}_{s}$, and $z_{4} \in \bar{L}_{s}$, then $L_{s}$ and $\bar{L}_{s-1}$ are under $\mathbf{C}_{4}$ configuration;

- if $z_{1} \notin L_{s}$, then $L_{s}$ and $\bar{L}_{s-1}$ are under $\mathbf{C}_{\mathbf{1}}, \mathbf{C}_{\mathbf{2}}$ or $\mathbf{C}_{\mathbf{3}}$ configuration (we could have to invert the roles of $L_{s}$ and $\bar{L}_{s}$ ).

If $e_{s}=2$ and if the lists $L_{s}$ and $\bar{L}_{s}$ are not under $\mathbf{C}_{\mathbf{1}}-\mathbf{C}_{\mathbf{4}}$ configurations, we have two cases to consider.

In the first case, there exists some subscripts $0 \leq t<s$ and $0 \leq t^{\prime}<s^{\prime}$ such that $x_{t}=x_{s}$ and $x_{t^{\prime}}=x_{s^{\prime}}$; if $s^{\prime}<s$ we set $i=t^{\prime}$ and $p=s^{\prime}$, if $s^{\prime}=s$, we set $i=\min \left(t, t^{\prime}\right)$ and $p=\max \left(t, t^{\prime}\right)$. As noticed above, when we go from lists $L_{s}$ and $\bar{L}_{s}$ to the lists $L_{s+1}$ and $\bar{L}_{s+1}$ we gain 0,1 or two equalities. Denote by $\sigma$ the least subscript greater than $s$ such that there exist 3 or 4 equalities among the elements of $L_{\sigma}$ and $\bar{L}_{\sigma}$; if such a subscript fails to exist, the lists $L_{2 n}$ and $\bar{L}_{2 n}$ are under $\mathbf{C}_{\mathbf{0}}$ configuration. Otherwise, assume that there exists exactly three equalities between the elements of $L_{\sigma} \cup \bar{L}_{\sigma}$, this additional equality can be one of the followings

- $\bar{x}_{\sigma}=x_{j}$ (with $j<\sigma$ ), the lists $L_{2 n}$ and $\bar{L}_{2 n}$ are then under $\mathbf{C}_{\mathbf{1}}$ configuration;

- $\bar{x}_{\sigma}=\bar{x}_{j}$ (with $j<\sigma$ ), the lists $L_{p}$ and $\bar{L}_{\sigma}$ are under $\mathbf{C}_{4}$ configuration;

- $x_{\sigma}=\bar{x}_{m}$ (with $m<\sigma$ ), the lists $L_{2 n}$ and $\bar{L}_{2 n}$ are then under $\mathbf{C}_{\mathbf{1}}$ configuration;

- $x_{\sigma}=x_{j}$ in this case we seek for the least subscript $\sigma^{\prime}>\sigma$ (if any) such that one get 1 or 2 additional equalities by going from $L_{\sigma} \cup \bar{L}_{\sigma}$ to $L_{\sigma^{\prime}} \cup \bar{L}_{\sigma^{\prime}}$.

If we are in the case where there exist exactly 4 equalities between the elements of $L_{\sigma} \cup \bar{L}_{\sigma}$, these equalities can be

- $x_{\sigma}=x_{j_{1}}$ and $\bar{x}_{\sigma}=x_{j_{2}}$ (with $j_{1}, j_{2}<\sigma$ ), in this case the lists $L_{2 n}$ and $\bar{L}_{2 n}$ are then under $\mathbf{C}_{\mathbf{1}}$ configuration;

- $x_{\sigma}=x_{j_{1}}$ and $\bar{x}_{\sigma}=\bar{x}_{j_{2}}$, the lists $L_{p}$ and $\bar{L}_{\sigma}$ are then under $\mathbf{C}_{\mathbf{4}}$ configuration;

- $x_{\sigma}=\bar{x}_{j_{1}}$ and $\bar{x}_{\sigma}=x_{j_{2}}$, the lists $L_{p}$ and $\bar{L}_{j_{1}}$ are then under $\mathbf{C}_{\mathbf{1}}$ configuration;

- $x_{\sigma}=\bar{x}_{j_{1}}$ and $\bar{x}_{\sigma}=\bar{x}_{j_{2}}$, the lists $L_{p}$ and $\bar{L}_{\sigma}$ are then under $\mathbf{C}_{4}$ configuration. In the second case there exist some subscripts $0 \leq t<s$ and $0 \leq t^{\prime}<s^{\prime}<s$ such that $s-t=s^{\prime}-t^{\prime}, x_{s}=\bar{x}_{t}$ and $x_{s^{\prime}}=\bar{x}_{t^{\prime}}$ (without loss of generality, we can exchange the roles of $x$ and $\bar{x}$ ). Proceeding as in the first case, we prove that either the lists $L_{2 n}$ and $\bar{L}_{2 n}$ are under $\mathbf{C}_{\mathbf{0}}$ configuration or there exists a subscript $s<\sigma \leq 2 n$ such that $L_{\sigma}$ and $\bar{L}_{\sigma}$ are under one of the configurations $\mathbf{C}_{\mathbf{1}}, \mathbf{C}_{\mathbf{2}}$ or $\mathbf{C}_{\mathbf{3}}$.

Let the pair $(f, h) \in \Gamma_{U}(X) \times C^{\infty}\left(X, \mathbf{R}^{p}\right)$ be fixed; hereafter, we shall say that the configuration of the triplet $\left(x, \bar{x}, \underline{u_{2 n}}\right)(x \neq \bar{x})$ is $\mathbf{C}_{\boldsymbol{i}}$ if the configuration of the lists $L_{2 n}$ and $\bar{L}_{2 n}$ related to $x$ and $\bar{x}$ is $\mathbf{C}_{\boldsymbol{i}}$. In the sequel, we shall assume that all the function spaces (such that $C^{\infty}\left(X, \mathbf{R}^{p}\right), \ldots$ ) as well as the spaces $\Gamma(X), \Gamma_{U}(X)$ and $\mathscr{G}_{2}^{U}(a)$ are endowed with the $C^{r}$ topology, where $r \in \mathbf{N}^{*}$. 
2.2. Outline of the proof of Theorem 4. Without going into the technique details, we shall explain our strategy for the proof. Take $(f, h) \in \Gamma_{U}(X) \times$ $C^{\infty}\left(X, \mathbf{R}^{d_{y}}\right)$, let $x_{0} \neq \bar{x}_{0}$ be two different initial conditions and $\underline{u_{2 n+1}}$ a sequence of controls. The equality $\Theta_{T}^{f, h}\left(x_{0}, u_{2 n+1}\right)=\Theta_{T}^{f, h}\left(\bar{x}_{0}, u_{2 n+1}\right)$ can be formulated in geometric terms. To show where are the difficulties, we make a first attempt by considering the simplest way to make this formulation. Consider the mapping, denoted by $r_{f, h}$, related to the pair $(f, h)$ and defined as

$$
\begin{aligned}
r_{f, h}: \quad X^{(2)} \times U^{2 n} & \longrightarrow \mathbf{R}^{(2 n+1) d_{y}} \\
\left(x, \bar{x}, \underline{u_{2 n+1}}\right) & \longmapsto\left(y_{0}-\bar{y}_{0}, \ldots, y_{2 n}-\bar{y}_{2 n}\right)
\end{aligned}
$$

where $y_{i}=h\left(x_{i}\right), \bar{y}_{i}=f\left(\bar{x}_{i}\right)$ and the $x_{i}$ 's and the $\bar{x}_{i}$ 's defined as in (3). The equality $\Theta_{T}^{f, h}\left(x_{0}, \underline{u_{2 n+1}}\right)=\Theta_{T}^{f, h}\left(\bar{x}_{0}, \underline{u_{2 n+1}}\right)$ means that $r_{f, h}\left(x_{0}, \bar{x}_{0}, \underline{u_{2 n}}\right)$ belongs to

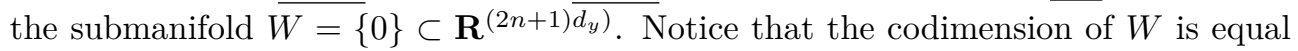
to $(2 n+1) d_{y}$ and is greater than $2 n\left(d_{u}+1\right)$ the dimension of the domain of $r_{f, h}$. If $r_{f, h}$ is transverse to $W$, this inequality on codim $W$ implies that $r_{f, h}\left(x_{0}, \bar{x}_{0}, \underline{u_{2 n}}\right)$ does not belong to $W$ and therefore that $\Theta_{T}^{f, h}\left(x_{0}, u_{2 n+1}\right) \neq \Theta_{T}^{f, h}\left(\bar{x}_{0}, u_{2 n+1}\right)$. Assume now

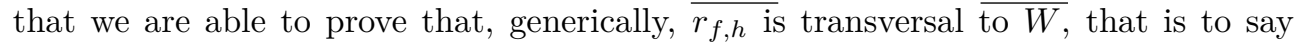
assume that there exists a residual set $\mathscr{R}$ such that $r_{f, h}$ is transversal to $W$ whenever $(f, h)$ belongs to $\mathscr{R}$, we then have proved that, generically, $\Theta_{T}^{f, h}$ is one-to-one. In order to prove that, generically, $r_{f, h}$ is transversal to $W$, we could try to apply Theorem 3 by proving that the evaluation map related to the representation $r$ from $\Gamma_{U}(X) \times$ $C^{\infty}\left(X, \mathbf{R}^{d_{y}}\right)$ to $C^{r}\left(X^{(2)} \times U^{2 n}, \mathbf{R}^{\left.(2 n+1) d_{y}\right)}\right.$ is transversal to $W$. This evaluation map $\mathrm{ev}_{r}$ is defined as $\operatorname{ev}_{r}\left(f, h, x, \bar{x}, \underline{u_{2 n}}\right)=r_{f, h}\left(x, \bar{x}, \underline{u_{2 n}}\right)$, as it is linear with respect to $h$, its differential with respect to $h$ is given by $\eta \mapsto\left(\eta\left(x_{0}\right)-\eta\left(\bar{x}_{0}\right), \ldots, \eta\left(x_{2 n}\right)-\right.$ $\left.\eta\left(\bar{x}_{2 n}\right)\right)$, if we were able to show that there exists $\eta$ in $C^{\infty}\left(X, \mathbf{R}^{d_{y}}\right)$ such that $\eta\left(x_{i}\right)-$ $\eta\left(\bar{x}_{i}\right)=\mathfrak{Y}_{i}$, for arbitrary vectors $\mathfrak{Y}_{0}, \ldots, \mathfrak{Y}_{2 n}$ of $\mathbf{R}^{d_{y}}$, then we would be done. The existence of such an $\eta$ is generally not ensured but is certainly true if the elements $x_{0}, \ldots, x_{2 n}, \bar{x}_{0}, \ldots, \bar{x}_{2 n}$ are all different or, more generally, if the two lists $L_{2 n}$ and $\bar{L}_{2 n}$ are under $\mathbf{C}_{0}$ configuration. In this case, modifying $r_{f, h}$ and the definition of $W$ as explained in the next section, we can prove that, generically $r_{f, h}$ is transversal to $W$. Now the two lists are not always under such a configuration, the points $x_{0}$ and $\bar{x}_{0}$ could be located on a periodic trajectory of $f$ or could be singular points. The case of singular points shows that we cannot disregard the cases where the two lists $L_{2 n}$ and $\bar{L}_{2 n}$ are not under configuration $\mathbf{C}_{\mathbf{0}}$. Assume that $x_{0}$ and $\bar{x}_{0}$ are singular points for the vector field $f\left(\cdot, u_{0}\right)$ and take a sequence of identical controls: $u_{0}=u_{1}=\ldots,=u_{2 n}$, then we cannot argue as in the case of $\mathbf{C}_{\mathbf{0}}$ configuration: a mapping $\eta$ as above fails to exist because $x_{0}=\cdots=x_{2 n}$ and $\bar{x}_{0}=\ldots \bar{x}_{2 n}$. Notice that this situation is unavoidable: on some manifolds, every vector field has at least one singular point; this means that the particular configurations $\mathbf{C}_{\mathbf{1}}-\mathbf{C}_{\mathbf{4}}$ cannot be eliminated by using an argument of density.

The outline of the rest of this section will be the following: for each configuration $\mathbf{C}_{0}-\mathbf{C}_{4}$, we shall prove that there exists a residual subset of $\Gamma_{U}(X) \times C^{r}\left(X, \mathbf{R}^{d_{y}}\right)$ (endowed with the $C^{r}$ topology), denoted by $\mathscr{C}_{k}^{r}(k=0, \ldots, 4)$, such that if $(f, h) \in$ $\mathscr{C}_{k}^{r}$ and if $\left(x_{0}, \bar{x}_{0}, \underline{u_{2 n}}\right)$ is in configuration $\mathbf{C}_{\mathbf{k}}$, then $\Theta_{T}^{f, h}\left(x_{0}, \underline{u_{2 n}}\right) \neq \Theta_{T}^{f, h}\left(\bar{x}_{0}, \underline{u_{2 n}}\right)$. Consider the intersection $\mathscr{C}^{r} \triangleq \mathscr{C}_{0}^{r} \cap \cdots \cap \mathscr{C}_{4}^{r}$, which also is a residual subset, and take a pair $(f, h)$ in $\mathscr{C}^{r}$; let $x_{0} \neq \bar{x}_{0}$ be two different initial conditions and $u_{2 n}$ a finite sequence of controls, as $\left(x_{0}, \bar{x}_{0}, u_{2 n}\right)$ must be in one of the $\mathbf{C}_{\mathbf{0}}-\mathbf{C}_{\mathbf{4}}$ configurations, we have $\Theta_{T}^{f, h}\left(x_{0}, \underline{u_{2 n}}\right) \neq \Theta_{T}^{f, h}\left(\bar{x}_{0}, \underline{u_{2 n}}\right)$. Now taking the intersections of the sets $\mathscr{C}^{r}$ for 
$r=1,2 \ldots$, we obtain a residual $\mathscr{R}$ of $\Gamma_{U}(X) \times C^{r}\left(X, \mathbf{R}^{d_{y}}\right)$ endowed with the $C^{\infty}$ topology; this residual is such that if $(f, h)$ belongs to $\mathscr{R}$, then $\Theta_{T}^{f, h}$ is one-to-one.

In the following section, the existence of the residual sets $\mathscr{C}_{0}^{r}-\mathscr{C}_{4}^{r}$ is stated in propositions 5, 9 and 10. For the proofs of these propositions, our strategy will be the following: we shall consider some submanifold $W$ together with some representation $\rho$; the choice of $W$ and $\rho$ being related to the considered configuration of the lists $L_{2 n}$ and $\bar{L}_{2 n}$. Concerning $W$ and $\rho$, we shall prove the following results:

- by applying the Transversal density theorem [1], we shall see that the set of pairs $(f, h) \in \Gamma_{U}(X) \times C^{\infty}\left(X, \mathbf{R}^{d_{y}}\right)$ which are transversal to $W$ is dense;

- we shall prove also that the codimension of $W$ is greater than the dimension of the domain of $\rho_{f, h}$, which implies that the range of $\rho_{f, h}$ does not intersect $W$;

- due to our choice of $W$ saying that $\rho_{f, h}\left(x, \bar{x}, \underline{u_{2 n}}\right) \notin W$ will imply that $\Theta_{T}^{f, h}\left(x, u_{2 n}\right) \neq \Theta_{T}^{f, h}\left(\bar{x}, u_{2 n}\right)$.

We shall provide a detailed proof for the $\mathbf{C}_{\mathbf{0}}$ and $\mathbf{C}_{\mathbf{1}}$ configurations, the proofs for the $\mathbf{C}_{\mathbf{2}}$ and $\mathbf{C}_{\mathbf{3}}$ configurations will be omitted because they are very similar to the previous ones. Concerning the configurations $\mathbf{C}_{\mathbf{0}}-\mathbf{C}_{\mathbf{3}}$, we only need the assumption $d_{y} \geq d_{u}+1$ to prove the existence of the residual subsets $\mathscr{C}_{0}-\mathscr{C}_{3}$. We have to consider apart the case of $\mathbf{C}_{4}$ configuration because to prove the existence of the residual subset $\mathscr{C}_{4}$ we need the assumption $d_{y} \geq d_{u}+2$.

3. The triplet $\left(x, \bar{x}, \underline{u_{2 n}}\right)$ is under configuration $\mathrm{C}_{0}$. In this section, we deal first with the simplest case: the $\mathbf{C}_{\mathbf{0}}$ configuration.

Proposition 5. Assume that $d_{y}>d_{u}$ (the number of observations is greater than the number of controls). Denote by $\mathscr{C}_{0}^{r}$ the set of pairs $(f, h) \in \Gamma_{U}(X) \times C^{\infty}\left(X, \mathbf{R}^{d_{y}}\right)$ such that $\Theta_{T}^{f, h}\left(x, u_{2 n+1}\right) \neq \Theta_{T}^{f, h}\left(\bar{x}, u_{2 n+1}\right)$ whenever the triplet $\left(x, \bar{x}, \underline{u_{2 n}}\right)($ with $x \neq \bar{x})$ is in configuration $\mathbf{C}_{\mathbf{0}}$. Then $\mathscr{C}_{0}^{r}$ contains a residual for the $C^{r}$ topology.

Proof. We consider the representation

$$
\begin{aligned}
\rho: \quad \Gamma_{U}(X) \times C^{\infty}\left(X, \mathbf{R}^{d_{y}}\right) & \longrightarrow C^{r}\left(X^{(2)} \times U^{2 n},\left(X^{2 n+1}\right)^{2} \times \mathbf{R}^{(2 n+1) d_{y}}\right) \\
(f, h) & \longmapsto \rho_{f, h}
\end{aligned}
$$

where $\rho_{f, h}$ is the mapping

$$
\begin{aligned}
\rho_{f, h}: X^{(2)} \times U^{2 n} & \longrightarrow X^{2 n+1} \times X^{2 n+1} \times \mathbf{R}^{(2 n+1) d_{y}} \\
\left(x, \bar{x}, \underline{u_{2 n}}\right) & \longmapsto\left(x_{0}, \ldots, x_{2 n}, \bar{x}_{0}, \ldots, \bar{x}_{2 n}, y_{0}-\bar{y}_{0}, \ldots, y_{2 n}-\bar{y}_{2 n}\right),
\end{aligned}
$$

and where $y_{i}=h\left(x_{i}\right)$ and $\bar{y}_{i}=h\left(\bar{x}_{i}\right)$, the $x_{i}$ 's and the $\bar{x}_{i}$ 's being defined above. We consider the submanifold $W_{0}$ included in $\left(X^{2 n+1}\right)^{2} \times \mathbf{R}^{(2 n+1) d_{y}}$ defined as follows. Submanifold $W_{0}$ is the set of those elements $\left(a_{0}, \ldots, a_{2 n}, \bar{a}_{0}, \ldots, \bar{a}_{2 n}, 0, \ldots, 0\right)$ such that

- the elements $\bar{a}_{0}, \ldots, \bar{a}_{2 n}$ are pairwise distinct;

- we have $a_{k} \neq \bar{a}_{l}$ if $k>l$.

Notice that the codimension of $W_{0}$ is equal to $(2 n+1) d_{y}$, as $d_{y}>d_{u}$, we have $(2 n+1) d_{y} \geq(2 n+1) d_{u}+2 n+1$, so the codimension of $W_{0}$ is greater than the dimension of $X^{(2)} \times U^{2 n+1}$, the domain of $\rho_{f, h}$.

Recall that the evaluation map $\mathrm{ev}_{\rho}$ is defined as:

$$
\operatorname{ev}_{\rho}\left(f, h, x, \bar{x}, \underline{u_{2 n}}\right)=\rho_{f, h}\left(x, \bar{x}, \underline{u_{2 n}}\right) .
$$


We shall see that $\mathrm{ev}_{\rho}$ is transversal to $W_{0}$ at every given point

$$
\mathscr{X} \triangleq\left(f, h, x, \bar{x}, \underline{u_{2 n}}\right) \in \Gamma_{U}(X) \times C^{r}\left(X, \mathbf{R}^{d_{y}}\right) \times X^{(2)} \times U^{2 n} .
$$

Consider a point $\mathscr{X}$ such that $\operatorname{ev}_{\rho}(\mathscr{X}) \in W_{0}$ and a vector $(\mathfrak{X}, \overline{\mathfrak{X}}, \mathfrak{Y})$ that is tangent to the codomain of $\mathrm{ev}_{\rho}$, with $\mathfrak{X}_{i} \in \mathrm{T}_{x_{i}} X, \overline{\mathfrak{X}}_{i} \in \mathrm{T}_{\bar{x}_{i}} X$, and $\mathfrak{Y}_{i} \in \mathbf{R}^{d_{y}}(i=0, \ldots, 2 n)$; we have to prove that there exist $\phi \in \Gamma_{U}(X), \eta \in C^{\infty}\left(X, \mathbf{R}^{d_{y}}\right), \xi \in \mathrm{T}_{x} X, \bar{\xi} \in \mathrm{T}_{\bar{x}} X$, $\nu_{i} \in \mathrm{T}_{u_{i}} U$ (for $\left.i=0, \ldots, 2 n\right)$ and a vector $\zeta$ in the tangent space to $W_{0}$ at $\operatorname{ev}_{\rho}(\mathscr{X})$ such that

$$
(\mathfrak{X}, \overline{\mathfrak{X}}, \mathfrak{Y})=\mathrm{d}\left(\operatorname{ev}_{\rho}\right)(\mathscr{X}) \cdot(\phi, \eta, \xi, \bar{\xi}, \nu)+\zeta .
$$

We shall prove this relation with $\phi=0, \xi=0, \bar{\xi}=0$ and $\nu=0$. We denote by

$$
\alpha_{0}, \ldots, \alpha_{2 n}, \bar{\alpha}_{0}, \ldots, \bar{\alpha}_{2 n}, 0, \ldots, 0
$$

the components of $\zeta$. In the right-hand member of (5), the 2(2n+1) first components are equal to $\alpha_{0}, \ldots, \alpha_{2 n}, \bar{\alpha}_{0}, \ldots, \bar{\alpha}_{2 n}$, and can be chosen such that $\alpha_{i}=\mathfrak{X}_{i}$ and $\bar{\alpha}_{i}=\overline{\mathfrak{X}}_{i}$ $(i=0, \ldots, 2 n)$. The $2 n+1$ last equations in (5) are

$$
\eta_{0}-\bar{\eta}_{0}=\mathfrak{Y}_{0}, \quad \ldots, \quad \eta_{2 n}-\bar{\eta}_{2 n}=\mathfrak{Y}_{2 n}
$$

where we let $\eta_{i} \triangleq \eta\left(x_{i}\right)$ and $\bar{\eta}_{i} \triangleq \eta\left(\bar{x}_{i}\right)$. We consider this system as a linear system whose unknown are $\eta_{0}, \ldots, \eta_{2 n}, \bar{\eta}_{0}, \ldots, \bar{\eta}_{2 n}$. If $\operatorname{ev}_{\rho}(\mathscr{X})$ belongs to the submanifold $W_{0}$, the points $\bar{x}_{0}, \ldots, \bar{x}_{2 n}$ are pairwise distinct, so the unknown $\bar{\eta}_{0}, \ldots, \bar{\eta}_{2 n}$ can be arbitrarily and independently chosen. There could be some equalities between the elements of the list $L_{2 n}$ and between an element of $L_{2 n}$ and an element of $\bar{L}_{2 n}$. If an equality such that $x_{k}=\bar{x}_{l}$ exists, then, as $\operatorname{ev}_{\rho}(\mathscr{X}) \in W_{0}$, we necessarily have $k<l$ and $\eta_{k}=\bar{\eta}_{l}$. The matrix of system (6) is then

$$
M=\left(\begin{array}{lll}
A & \mid & -I_{(2 n+1) d_{y}}+B
\end{array}\right)
$$

Where $I_{(2 n+1) d_{y}}$ is the $(2 n+1) d_{y}$ dimensional identity matrix and matrix $B$ is a block matrix, whose blocks are 0 or $d_{y}$ dimensional identity matrices. Moreover, matrix $B$ is upper triangular: if we have an equality like $x_{k}=\bar{x}_{l}$, then we find in $B$ the block $I_{d_{y}}$ (identity matrix) at position $(k, l)$ with $l>k$. From the form of matrix $B$, we can conclude that the rank of $M$ is equal to $(2 n+1) d_{y}$ which implies that we can find $\bar{\eta}_{0}, \ldots, \bar{\eta}_{2 n}, \eta_{0}, \ldots, \eta_{2 n}$ such that system (6) has a solution. Denote by $x_{k_{1}}, \ldots, x_{k_{p}}$ the list of the elements of $L_{2 n}$ which are not equal to an element of $\bar{L}_{2 n}$. It is possible to find a solution of (6) such that $\eta_{k_{1}}=\cdots=\eta_{k_{p}}=0$, for such a solution, it is then possible to find a mapping $\eta$ such that $\eta\left(x_{i}\right)=\eta_{i}$ and $\eta\left(\bar{x}_{i}\right)=\bar{\eta}_{i}(i=0, \ldots, 2 n)$.

We have shown that $\mathrm{ev}_{\rho}$ is transversal to $W_{0}$. The conclusion of the proposition now follows from the application of the Transversal density theorem [1]: the set $\mathscr{O}_{0}^{r}$ of pairs $(f, h) \in \Gamma_{U}(X) \times C^{\infty}\left(X, \mathbf{R}^{d_{y}}\right)$ such that $\rho_{f, h}$ is transversal to $W_{0}$ is open and dense in the $C^{r}$ topology (for every $r>0$ ). Take now a pair $(f, h)$ in this set $\mathscr{O}_{0}^{r}$ and take two initial conditions $x \neq \bar{x}$, and a finite sequence of controls $u_{2 n}$ such that the triplet $\left(x, \bar{x}, u_{2 n}\right)$ is in configuration $\mathbf{C}_{\mathbf{0}}$, the mapping $\rho_{f, h}$ is transversal to $W_{0}$ at $\left(x, \bar{x}, u_{2 n}\right)$ but as codim $W_{0}>\operatorname{dim}\left(X^{(2)} \times U^{2 n}\right)$, transversality means that $\rho_{f, h}\left(x, \bar{x}, u_{2 n}\right) \notin W_{0}$, which implies that at least one of the equalities $y_{j}=\bar{y}_{j}$ is not satisfied and so $\Theta_{T}^{f, h}\left(x, \underline{u_{2 n}}\right) \neq \Theta_{T}^{f, h}\left(\bar{x}, \underline{u_{2 n}}\right)$. 
4. The case of the $\mathbf{C}_{1}-\mathbf{C}_{4}$ configurations. In the proofs of the next propositions 9 and 10, we shall have to consider the derivative of $\varphi_{T}(x)$ with respect to the vector field $f$ (we are no more able to take $\phi=0$ as in the proof of Proposition 5). This is why we ned to state a technical lemma, which bears on the computation of these derivatives. To prove this lemma, we have to take into account the periodic trajectories of a vector field; these trajectories have some generic properties - which we intend to use in the proof of Lemma 8-, which are stated in the Kupka-Smale theorem. Now the Kupka-Smale theorem has not been stated for parametrized vector fields, so we will show that it can be generalized to those vector fields.

4.1. Periodic trajectories and the Kupka-Smale theorem. Take a parametrized vector field $f \in \Gamma_{U}(X), u \in U, x \in X$, and assume that $x$ belongs to a periodic trajectory of the vector field $f(\cdot, u)$. Then there exists $\pi_{0}>0$ such that $\varphi_{\pi_{0}}^{u}(x)=x$, this implies that $\mathrm{d} \varphi_{\pi_{0}}^{u}(x) \cdot f(x, u)=f(x, u)$, so 1 is an eigenvalue of $A \triangleq \mathrm{d} \varphi_{\pi_{0}}^{u}(x)$. In the sequel we shall have to consider expressions like $\mathrm{Id}+A+\cdots+A^{k}$ and we shall need that this sum of linear mappings be invertible; this is certainly true if, apart from 1, the other eigenvalues have modulus different from 1. The theorem of KupkaSmale $[15,17]$ asserts that this is generically the case for a vector field. Let $a>0$, hereafter, we denote by $\mathscr{G}_{2}(a)$ the subset of $\Gamma(X)$ of those vector fields $f$ such that

- if $x$ is a singular point of $f(i . e f(x)=0)$, then for every $t \neq 0, \mathrm{~d} \varphi_{t}(x)$ : $\mathrm{T}_{x} X \rightarrow \mathrm{T}_{x} X$ has no complex eigenvalue of modulus 1 ;

- if $x$ belongs to a periodic trajectory of $f$ with period $0<\pi_{0} \leq a$, then, denoting by $1, \lambda_{2}, \ldots, \lambda_{n}$ the eigenvalues of $\mathrm{d} \varphi_{\pi_{0}}(x)$, we have $\left|\lambda_{i}\right| \neq 1$ for $i=2, \ldots, n$.

Hereafter, recall that the manifolds $X$ and $U$ are assumed to be compact. We have

TheOREM 6 (Kupka-Smale). Let $a>0$, the set $\mathscr{G}_{2}(a)$ is residual; moreover for the $C^{r}$ topology $(r<+\infty), \mathscr{G}_{2}(a)$ is open and dense.

This theorem can be generalized to parametrized vector fields, namely we have.

THEOREM 7. Let $a>0$, the set $\mathscr{G}_{2}^{U}(a)$ of parametrized vector fields such that $f(\cdot, u) \in \mathscr{G}_{2}(a)$ for every $u \in U$ is a residual; moreover $\mathscr{G}_{2}^{U}(a)$ is open and dense for the $C^{r}$ topology.

This theorem can be proved by adapting the steps of the proof of the KupkaSmale's theorem which can be found in [1]. Owing to lack of space, we do not write here the proof of Theorem 7 but we give a sketch of this proof in Appendix A; moreover, the reader is referred to [19] where this result is proved in the case where the dimension of $U$ is 1 .

4.2. Technical lemma. The proofs of propositions 9 and 10 below will follow the same scheme as the proof of Prop. 5. Nevertheless, in the following propositions, in order to prove that the mapping $\mathrm{ev}_{\rho}$ is transversal to some submanifold $W$, we shall have to consider the derivative of the flow with respect to a vector field. Thus, before going further, we recall a result which will be used in the proof of Lemma 8 and propositions 9 and 10. Take two vector fields $f$ and $\phi$ defined on $X$ and denote by $\varphi_{t}$ and $\varphi_{t}^{\lambda}(\lambda \in \mathbf{R})$ the flows related to $f$ and $f+\lambda \phi$ respectively. In [1, Perturbation theorem], the following formula is proved: for every $x \in X$, we have

$$
\left.\frac{\mathrm{d}}{\mathrm{d} \lambda} \varphi_{t}^{\lambda}(x)\right|_{\lambda=0}=\int_{0}^{t} \mathrm{~d} \varphi_{\sigma} \circ \phi \circ \varphi_{t-\sigma}(x) \mathrm{d} \sigma .
$$


Obviously, this formula can be extended to the case of parametrized vector fields. Consider $f$ and $\phi$ in $\Gamma_{U}(X)$ and denote by $\varphi_{t}^{u, \lambda}$ the flow generated by the vector field $f(\cdot, u)+\lambda \phi(\cdot, u)$ (with $u$ fixed). Starting from an initial condition $x_{0}$, consider now the sequence $x_{0}^{\lambda}, x_{1}^{\lambda}, \ldots$ defined recursively as $x_{0}^{\lambda}=x_{0}$ and $x_{i+1}^{\lambda}=\varphi_{T}^{u_{i}, \lambda}\left(x_{i}^{\lambda}\right)$, then applying formula (7), we deduce easily that

$$
\left.\frac{\mathrm{d}}{\mathrm{d} \lambda} x_{i+1}^{\lambda}\right|_{\lambda=0}=J_{i}+\delta_{i}\left(J_{i-1}\right)+\cdots+\delta_{1}\left(J_{0}\right)
$$

where

$$
J_{k}=\int_{0}^{T} \mathrm{~d} \varphi_{\sigma}^{u_{k}}\left(\varphi_{T-\sigma}^{u_{k}}\left(x_{k}\right)\right) \cdot \phi\left(\varphi_{T-\sigma}^{u_{k}}\left(x_{k}\right), u_{k}\right) \mathrm{d} \sigma
$$

the integral $J_{k}$ belongs to the tangent space of $X$ at $\varphi_{T}^{u_{k}}\left(x_{k}\right)=x_{k+1}$. Moreover the $\delta_{k}$ 's are the mappings defined as

$$
\delta_{k}=\mathrm{d}\left(\varphi_{T}^{u_{i}} \circ \cdots \circ \varphi_{T}^{u_{k}}\right)\left(x_{k}\right) .
$$

We state now a preliminary result which will be used in the proofs of propositions 9 and 10 . We shall say that these lists are under $\mathbf{C}_{\mathbf{3}}^{\prime}$ (resp. $\mathbf{C}_{\mathbf{4}}^{\prime}$ ) configuration if

- they are under $\mathbf{C}_{\mathbf{3}}$ (resp. $\mathbf{C}_{4}$ ) configuration and if

- there exists a subscript $k \in\{i, \ldots, p-1\} \cup\{m, \ldots, j-1\}$ (resp. $k \in\{i, \ldots, p-$ $1\} \cup\{j, \ldots, m-1\})$ such that $u_{k} \neq u_{p-1}$.

The proof of the following lemma is postponed in Appendix B.

Lemma 8. Let $\mathfrak{X}_{p} \in \mathrm{T}_{x_{p}} X$ be an arbitrary tangent vector to $X$ at $x_{p}$. Assume that the lists $L_{2 n}$ and $\bar{L}_{2 n}$ are under $\mathbf{C}_{\mathbf{1}}$ configuration, then one can find a vector field $\phi \in \Gamma_{U}(X)$ such that we have

$$
\left.\frac{\mathrm{d} x_{p}^{\lambda}}{\mathrm{d} \lambda}\right|_{\lambda=0}=\mathfrak{X}_{p},\left.\quad \frac{\mathrm{d} x_{i}^{\lambda}}{\mathrm{d} \lambda}\right|_{\lambda=0}=0 .
$$

If these lists are under $\mathbf{C}_{\mathbf{2}}, \mathbf{C}_{\mathbf{3}}^{\prime}$ or $\mathbf{C}_{\mathbf{4}}^{\prime}$ configuration, then one can find a vector field $\phi \in \Gamma_{U}(X)$ such that we have

$$
\left.\frac{\mathrm{d} x_{p}^{\lambda}}{\mathrm{d} \lambda}\right|_{\lambda=0}=\mathfrak{X}_{p},\left.\quad \frac{\mathrm{d} x_{i}^{\lambda}}{\mathrm{d} \lambda}\right|_{\lambda=0}=0,\left.\quad \frac{\mathrm{d} \bar{x}_{m}^{\lambda}}{\mathrm{d} \lambda}\right|_{\lambda=0}=0,\left.\quad \frac{\mathrm{d} \bar{x}_{j}^{\lambda}}{\mathrm{d} \lambda}\right|_{\lambda=0}=0 .
$$

4.3. The triplet $\left(x, \bar{x}, \underline{u_{2 n}}\right)$ is under one of the configurations $\mathrm{C}_{1}, \mathrm{C}_{2}$ or $\mathrm{C}_{3}$.

Proposition 9. Assume that $d_{y}>d_{u}$ (the number of observations is greater than the number of controls). For $k=1,2,3$, denote by $\mathscr{C}_{k}^{r}$ the subset of pairs $(f, h) \in$ $\Gamma_{U}(X) \times C^{\infty}\left(X, \mathbf{R}^{p}\right)$ such that $\Theta_{T}^{f, h}\left(x, \underline{u_{2 n+1}}\right) \neq \Theta_{T}^{f, h}\left(\bar{x}, \underline{u_{2 n+1}}\right)$ whenever the triplet $\left(x, \bar{x}, \underline{u_{2 n}}\right)$ (with $x \neq \bar{x}$ ) is in configuration $\mathbf{C}_{\boldsymbol{k}}$. Then each subset $\mathscr{C}_{k}^{r}$ contains a residual for the $C^{r}$ topology.

Hereafter, we write the proof of this proposition only in the case of $\mathbf{C}_{\mathbf{1}}$ configuration, the case proofs for the other configurations being very similar.

4.3.1. Proof of the proposition in the case of $\mathbf{C}_{\mathbf{1}}$ configuration. If the triplet $\left(x, \bar{x}, \underline{u_{2 n}}\right)$ is in the $\mathbf{C}_{\mathbf{1}}$ configuration, there exist subscripts $i, j, p, m$ such that $x_{p}=x_{i}$ and $\overline{\bar{x}_{m}}=x_{j}$. 
We choose four subscripts $0 \leq i, j, p, m \leq 2 n$ such that $i<p$; letting $q=$ $\max (i, j, p, m)$, we consider the representation $\rho$

$$
\begin{aligned}
\rho: \mathscr{G}_{2}^{U}(a) \times C^{r}\left(X, \mathbf{R}^{d_{y}}\right) & \longrightarrow C^{r}\left(X^{(2)} \times U^{q}, X^{q+1} \times X^{q+1} \times \mathbf{R}^{q d_{y}}\right) \\
(f, h) & \longmapsto \rho_{f, h}
\end{aligned}
$$

defined through the mapping $\rho_{f, h}$

$$
\begin{aligned}
& \rho_{f, h}: \quad X^{(2)} \times U^{q} \quad \longrightarrow X^{q+1} \times X^{q+1} \times \mathbf{R}^{q d_{y}} \\
& \left(x, \bar{x}, u_{q}\right) \longmapsto\left(x_{0}, \ldots, x_{q}, \bar{x}_{0}, \ldots, \bar{x}_{q}, y_{0}-\bar{y}_{0}, \ldots, y_{q-1}-\bar{y}_{q-1}\right) .
\end{aligned}
$$

We fix two lists of subscripts (possibly empty) $i_{1}<\cdots<i_{r}<q$ and $j_{1}<\cdots<j_{r}<q$ such that the signs of $i_{1}-j_{1}, \ldots, i_{r}-j_{r}$ are the same ; if $j, m<q$, one has $i_{k}=j$ and $j_{k}=m$ for some subscript $k$. We consider also the submanifold

$$
V_{m}^{j, i, p} \subset X^{q+1} \times X^{q+1} \times \mathbf{R}^{(2 n+1) d_{y}}
$$

defined as follows: $V_{m}^{j, i, p}$ is the set of those elements

$$
\left(a_{0}, \ldots, a_{q}, \bar{a}_{0}, \ldots, \bar{a}_{q}, 0, \ldots, 0\right)
$$

such that

- we have the equalities $\bar{a}_{m}=a_{j}$ and $a_{i}=a_{p}$;

- the elements of $\left\{\bar{a}_{0}, \ldots, \bar{a}_{q-1}\right\}$ are pairwise different;

- $a_{i^{\prime}} \neq \bar{a}_{j^{\prime}}$ if $\left(i^{\prime}, j^{\prime}\right) \neq\left(i_{k}, j_{k}\right)\left(i^{\prime}, j^{\prime}<q, k=1, \ldots, r\right)$.

Notice that the number of submanifold having the above properties is finite, moreover the codimension of $V_{m}^{j, i, p}$ is equal to $2 n+q d_{y}$ and is greater or equal to $2 n+q\left(d_{u}+1\right)>$ $2 n+q d_{u}$; so the codimension of $V_{m}^{j, i, p}$ is greater than the dimension of the domain of $\rho_{f, h}$.

We shall show that $\mathrm{ev}_{\rho}$ is transversal to $V_{m}^{j, i, p}$. Let $\mathscr{X} \triangleq\left(f, h, x, \bar{x}, \underline{u_{q}}\right)$ be a point such that $\operatorname{ev}_{\rho}(\mathscr{X}) \in V_{m}^{j, i, p}$ and take a vector $(\mathfrak{X}, \overline{\mathfrak{X}}, \mathfrak{Y})$ which is tangent to the codomain of $\operatorname{ev}_{\rho}$ at $\operatorname{ev}_{\rho}(\mathscr{X})$, with $\mathfrak{X}_{k} \in T_{x_{k}} X, \overline{\mathfrak{X}}_{k} \in T_{\bar{x}_{k}} X(k=0, \ldots, q)$ and $\mathfrak{Y}_{k} \in \mathbf{R}^{d_{y}}$ $(k=0, \ldots, q-1)$. We have to prove that there exist $\phi \in \Gamma_{U}(X), \eta \in C^{r}\left(X, \mathbf{R}^{d_{y}}\right)$, $\xi \in \mathrm{T}_{x} X, \bar{\xi} \in \mathrm{T}_{\bar{x}} X, \nu_{l} \in \mathrm{T}_{u_{l}} U$ (for $l=0, \ldots, m-1$ ) and a vector $\zeta$ in the tangent space to $V_{m}^{j, i, p}$ at $\operatorname{ev}_{\rho}(\mathscr{X})$ such that

$$
(\mathfrak{X}, \overline{\mathfrak{X}}, \mathfrak{Y})=\mathrm{d}\left(\operatorname{ev}_{\rho}\right)(\mathscr{X}) \cdot(\phi, \eta, \xi, \bar{\xi}, \nu)+\zeta .
$$

We shall prove this relation with $\xi=0$ and $\nu=0$. We denote by

$$
\alpha_{0}, \ldots, \alpha_{q}, \bar{\alpha}_{0}, \ldots, \bar{\alpha}_{q}, \beta_{0}, \ldots, 0, \ldots, 0
$$

the components of $\zeta$.

Among the $2 q+2$ first equations in (8), the ones corresponding to subscripts different from $j, i$ and $p$ (first $q+1$ equations) or $m$ (last $q+1$ equations) are trivial because the corresponding tangent vectors $\alpha_{k}(k \neq j, i, p)$ and $\bar{\alpha}_{k}(k \neq m)$ can be arbitrarily chosen. Thus we focus on the following four equations

(9) $\quad \alpha_{j}+A_{j}=\mathfrak{X}_{j}, \quad \alpha_{i}+A_{i}=\mathfrak{X}_{i}, \quad \alpha_{p}+A_{p}=\mathfrak{X}_{p}, \quad \bar{\alpha}_{m}+\bar{A}_{m}+\bar{\xi}_{m}=\mathfrak{X}_{m}$.

Here $A_{l}$ (resp. $\left.\bar{A}_{l}\right), l=0, \ldots, \underline{q}$, denotes the derivative of $x_{l}^{\lambda}$ (resp. $\bar{x}_{l}$ ) with respect to $\lambda$ evaluated at $\lambda=0$ while $\bar{\xi}_{j}$ is defined recursively as

$$
\bar{\xi}_{0}=\bar{\xi}
$$$$
\bar{\xi}_{k+1}=\mathrm{d} \varphi_{T}^{u_{k}}\left(\bar{x}_{k}\right) \cdot \bar{\xi}_{k},
$$$$
k=0, \ldots, 2 n-1 .
$$ 
Notice also that, as $\zeta$ is a tangent vector to $W_{r}^{j, i, p}$, necessarily, $\alpha_{i}=\alpha_{p}$ and $\alpha_{j}=\bar{\alpha}_{m}$. As we are under $\mathbf{C}_{\mathbf{1}}$ configuration, we can apply the preliminary lemma 8, thus we can find a vector field $\phi$ such that $A_{i}=0$ while $A_{p}$ is equal to an arbitrary tangent vector in $\mathrm{T}_{x_{p}} X$ (notice that we cannot guarantee that $A_{j}=0$ because we do not know the position of $j$ with respect to $i$ and $p$ ). Taking into account that $\alpha_{p}=\alpha_{i}$ and $\bar{\alpha}_{m}=\alpha_{j}$, the equations (9) then rewrite

$$
\alpha_{j}+A_{j}=\mathfrak{X}_{j}, \quad \alpha_{i}=\mathfrak{X}_{i}, \quad \alpha_{i}+A_{p}=\mathfrak{X}_{p}, \quad \alpha_{j}+\bar{A}_{m}+\bar{\xi}_{m}=\mathfrak{X}_{m},
$$

a solution to system (11) is then

$$
\alpha_{i}=\mathfrak{X}_{\mathfrak{i}}, \quad A_{p}=\mathfrak{X}_{p}-\mathfrak{X}_{i}, \quad \alpha_{j}=\mathfrak{X}_{\mathfrak{j}}-A_{j}, \quad \bar{\xi}_{m}=\mathfrak{X}_{m}-\bar{A}_{m}-\mathfrak{X}_{j}+A_{j}
$$

Notice that, once $A_{p}$ has been chosen, $A_{j}$ and $\bar{A}_{m}$ are fixed (they depend on $\mathfrak{X}_{p}-\mathfrak{X}_{i}$ ), moreover $\bar{\xi}$ can be chosen in such a way that $\bar{\xi}_{m}$ is equal to an arbitrary tangent vector.

As for the last $2 q$ equations, they can be written

$$
\eta_{0}-\bar{\eta}_{0}-\bar{\chi}_{0}=\mathfrak{Y}_{0}, \quad \ldots, \quad \eta_{q-1}-\bar{\eta}_{q-1}-\bar{\chi}_{q-1}=\mathfrak{Y}_{q-1}
$$

where $\bar{\chi}_{k}=\mathrm{d} h\left(x_{k}\right) \cdot \bar{\xi}_{k}, \eta_{k}=\eta\left(x_{k}\right)$, and $\bar{\eta}_{k}=\eta\left(\bar{x}_{k}\right)(k=0, \ldots, q-1)$. We regard this system as a linear system. As $\bar{x}_{m}=x_{j}$ is the only equality between the $x_{k}$ 's and the $\bar{x}_{k}$ 's, we can consider that the unknowns for this system are $\eta_{0}, \ldots, \eta_{q-1}$ and $\bar{\eta}_{0}, \ldots, \bar{\eta}_{q-1}$; the matrix of this system then writes

$$
\left(\begin{array}{lll}
A & \mid & -\mathrm{I}_{q d_{y}}+B
\end{array}\right)
$$

here $\mathrm{I}_{q d_{y}}$ denotes the $q d_{y}$ dimensional identity matrix while $B$ is a $d_{y} \times d_{y}$ block matrix which is a strictly upper or strictly lower triangular matrix. Thus $\mathrm{I}_{q d_{y}}-B$ is an upper or lower-triangular block matrix, the elements of the diagonal being equal to the $d_{y} \times d_{y}$ identity matrix, hence $-\mathrm{I}_{q d_{y}}+B$ is non singular, which proves that system (12) admits a solution.

This proves that $\mathrm{ev}_{\rho}$ is transversal to $V_{m}^{j, i, p}$; we achieve the proof of Proposition 9 as the one of Proposition 5.

4.4. The triplet $\left(x, \bar{x}, \underline{u_{2 n}}\right)$ is under one configuration $\mathbf{C}_{4}$. We examine now the case of $\mathbf{C}_{4}$ configuration, in this case, the assumption $d_{y} \geq d_{u}+1$ is no more sufficient.

Proposition 10. Assume that $d_{u}=0$ and $d_{y} \geq 1$, or $d_{u}>0$ and $d_{y} \geq d_{u}+2$ (the number of observations is greater than the number of controls plus one). Denote by $\mathscr{C}_{4}^{r}$ the set of pairs $(f, h) \in \Gamma_{U}(X) \times C^{\infty}\left(X, \mathbf{R}^{p}\right)$ such that $\Theta_{T}^{f, h}\left(x, u_{2 n+1}\right) \neq$ $\Theta_{T}^{f, h}\left(\bar{x}, \underline{u_{2 n+1}}\right)$ whenever the triplet $\left(x, \bar{x}, \underline{u_{2 n}}\right)$ (with $\left.x \neq \bar{x}\right)$ is in configuration $\mathbf{C}_{\mathbf{4}}$. Then $\mathscr{C}_{4}^{r}$ contains a residual for the $C^{r}$ topology.

Proof of Proposition 10. In the sequel, we shall say that the lists $L_{2 n}$ and $\bar{L}_{2 n}$ are under $\mathbf{C}_{4}^{\prime \prime}$ configuration if they are under $\mathbf{C}_{\mathbf{4}}$ configuration but not under $\mathbf{C}_{\mathbf{4}}^{\prime}$ configuration. We shall consider these two subcases separately.

Configuration $\mathbf{C}_{\mathbf{4}}^{\prime}$. In this case there exist subscripts $0 \leq i<p \leq 2 n$ and $0 \leq$ $j<m \leq 2 n$ such that $x_{i}=x_{p}$ and $\bar{x}_{j}=\bar{x}_{m}$; moreover there exists a subscripts $k \in\{i, \ldots, p-1\} \cup\{j, \ldots, m-1\}$ such that $u_{k} \neq u_{p-1}$. Without loss of generality, we can assume that $m \geq p$. 
We choose four subscripts $0 \leq i<p \leq 2 n$ and $0 \leq j<m \leq 2 n(m \geq p)$, as well as a subscript $k_{0} \in\{i, \ldots, p-1\} \cup\{j, \ldots, m-1\}$. We consider the representation $\rho$ defined on $\mathscr{G}_{2}^{U}(a) \times C^{r}\left(X, \mathbf{R}^{d_{y}}\right)$ through the mapping $\rho_{f, h}$ as

$$
\begin{aligned}
\rho_{f, h}: X^{(2)} \times U_{\left(k_{0}, p-1\right)}^{m} & \longrightarrow X^{m+1} \times X^{m+1} \times \mathbf{R}^{m d_{y}} \\
\left(x, \bar{x}, \underline{u_{m}}\right) & \longmapsto\left(x_{0}, \ldots, x_{m}, \bar{x}_{0}, \ldots, \bar{x}_{m}, y_{0}-\bar{y}_{0}, \ldots, y_{m-1}-\bar{y}_{m-1}\right) .
\end{aligned}
$$

Together with $\rho$, we consider the submanifold

$$
Z_{j, m}^{i, p} \subset X^{m+1} \times X^{m+1} \times \mathbf{R}^{m d_{y}}
$$

defined as the set of those elements

$$
\left(a_{0}, \ldots, a_{m}, \bar{a}_{0}, \ldots, \bar{a}_{m}, 0, \ldots, 0\right)
$$

such that

- we have the equalities $a_{i}=a_{p}$ and $\bar{a}_{j}=\bar{a}_{m}$;

- the above equalities are the only ones between the elements of $L_{p} \cup \bar{L}_{m}$.

Notice first that the codimension of $Z_{j, m}^{i, p}$ is equal to $2 n+m d_{y}$ and is greater than $2 n+m d_{u}$ which is greater than the dimension of the domain of $\rho_{f, h}$.

We shall show that $\mathrm{ev}_{\rho}$ is transversal to $Z_{j, m}^{i, p}$. Let $\mathscr{X} \triangleq\left(f, h, x, \bar{x}, \underline{u_{m}}\right)$ be a point such that $\operatorname{ev}_{\rho}(\mathscr{X}) \in Z_{j, m}^{i, p}$ and take a tangent vector $(\mathfrak{X}, \overline{\mathfrak{X}}, \mathfrak{Y})$ with $\mathfrak{X}_{k} \in T_{x_{k}} X$, $\overline{\mathfrak{X}}_{k} \in T_{\bar{x}_{k}} X(k=0, \ldots, m)$, and $\mathfrak{Y}_{k} \in \mathbf{R}^{d_{y}}(k=0, \ldots, m-1)$. We have to prove that there exist $\phi \in \Gamma_{U}(X), \eta \in C^{r}\left(X, \mathbf{R}^{d_{y}}\right), \xi \in \mathrm{T}_{x} X, \bar{\xi} \in \mathrm{T}_{\bar{x}} X, \nu_{k} \in \mathrm{T}_{u_{k}} U$ (for $j=0, \ldots, m-1)$ and a vector $\zeta$ in the tangent space to $Z_{j, m}^{i, p}$ at $\operatorname{ev}_{\rho}(\mathscr{X})$ such that

$$
(\mathfrak{X}, \overline{\mathfrak{X}}, \mathfrak{Y})=\mathrm{d}\left(\mathrm{ev}_{\rho}\right)\left(f, h, x, \bar{x}, \underline{u_{m}}\right) \cdot(\phi, \eta, \xi, \bar{\xi}, \nu)+\zeta .
$$

We shall prove this relation with $\xi=0, \bar{\xi}=0$ and $\nu=0$. We denote by

$$
\alpha_{0}, \ldots, \alpha_{m}, \bar{\alpha}_{0}, \ldots, \bar{\alpha}_{m}, 0, \ldots, 0
$$

the components of $\zeta$; notice that, as $\zeta$ is a tangent vector to $Z_{j, m}^{i, p}$, we have $\alpha_{i}=\alpha_{p}$ and $\bar{\alpha}_{j}=\bar{\alpha}_{m}$.

To prove that Equation (13) admits a solution, the reasoning is analogous to the one made in the proofs of the previous propositions but here, we have to apply twice Lemma 8. Hereafter, given a vector field $\phi$ we denote by $x_{i}^{\lambda, \phi}$, the sequence defined recursively as follows

$$
x_{0}^{\lambda, \phi}=x_{0}, \quad x_{i+1}^{\lambda, \phi}=\varphi_{T}^{u_{i}, \lambda}\left(x_{i}^{\lambda, \phi}\right)
$$

where $\varphi_{t}^{u_{i}, \lambda}$ denotes the flow related to the vector field $f\left(\cdot, u_{i}\right)+\lambda \phi$. According to Lemma 8 , there exist a vector field $\phi_{0}$ such that the derivatives of $x_{i}^{\lambda, \phi_{0}}, \bar{x}_{j}^{\lambda, \phi_{0}}$ and $\bar{x}_{m}^{\lambda, \phi_{0}}$ with respect to $\lambda$ are all zero while the derivative of $x_{p}^{\lambda, \phi_{0}}$ can be arbitrarily chosen. As there exists $k_{0}$ such that $u_{k_{0}} \neq u_{p-1}$, we can also apply this lemma by replacing $u_{p-1}$ by $u_{k_{0}}$ in the lemma. For example, if $j \leq k_{0}<m$, and, assuming without loss of generality, that $k_{0}$ is the greatest subscript less than $m$ such that $u_{k_{0}} \neq u_{p-1}$, we deduce from Lemma 8 that there exists a vector field $\phi_{1}$ such that the derivatives of $x_{i}^{\lambda, \phi_{1}}, x_{p}^{\lambda, \phi_{1}}$ and $\bar{x}_{j}^{\lambda, \phi_{1}}$ with respect to $\lambda$ are all zero while the derivative 
of $\bar{x}_{k_{0}+1}^{\lambda, \phi_{1}}$ can be arbitrarily chosen. Noticing that

$$
\bar{x}_{m}^{\lambda, \phi_{1}}= \begin{cases}\bar{x}_{k_{0}+1}^{\lambda, \phi_{1}} & \text { if } k_{0}=m-1, \\ \varphi_{\left(m-k_{0}\right) T}^{u_{p-1}}\left(\bar{x}_{k_{0}}^{\lambda, \phi_{1}}\right) & \text { if } k_{0}<m-1 ;\end{cases}
$$

we see that the derivative of $\bar{x}_{m}^{\lambda, \phi_{1}}$ with respect to $\lambda$ can be arbitrarily chosen.

We chose now the vector field $\phi \in \Gamma_{U}(X)$ as follows

- $\phi\left(\cdot, u_{p-1}\right)=\phi_{0}$ and $\phi\left(\cdot, u_{k_{0}}\right)=\phi_{1}$;

- $\phi\left(\cdot, u_{k}\right) \equiv 0$ if $u_{k} \notin\left\{u_{p-1}, u_{k_{0}}\right\}$.

Clearly the derivatives of $x_{i}^{\lambda, \phi}$ and $\bar{x}_{j}^{\lambda, \phi}$ with respect to $\lambda$ are zero while the derivatives of $x_{p}^{\lambda, \phi}$ and $\bar{x}_{m}^{\lambda, \phi}$ can be arbitrarily chosen.

As in the previous configurations, as regard the first $2 m+2$ equations in (13), we have to consider only the four following ones

$$
\alpha_{i}+A_{i}=\mathfrak{X}_{i}, \quad \alpha_{p}+A_{p}=\mathfrak{X}_{p}, \quad \bar{\alpha}_{j}+\bar{A}_{j}=\overline{\mathfrak{X}}_{j}, \quad \bar{\alpha}_{m}+\bar{A}_{m}=\overline{\mathfrak{X}}_{m}
$$

where the $A_{k}$ 's (resp. the $\bar{A}_{k}$ 's) denote the derivatives of the $x_{k}^{\lambda, \phi}$ (resp. of the $\bar{x}_{k}^{\lambda, \phi}$ ) with respect to $\lambda$; so we have $A_{i}=0$ and $\bar{A}_{j}=0$. Notice also that, from the definition of $Z_{j, m}^{i, p}$, it follows that $\alpha_{i}=\alpha_{p}$ and $\bar{\alpha}_{j}=\bar{\alpha}_{m}$. Taking into account these equalities, the solution to the equations (14) is then

$$
\alpha_{i}=\mathfrak{X}_{i}, \quad A_{p}=\mathfrak{X}_{p}-\mathfrak{X}_{j}, \quad \bar{\alpha}_{j}=\overline{\mathfrak{X}}_{j}, \quad \bar{A}_{m}=\overline{\mathfrak{X}}_{m}-\overline{\mathfrak{X}}_{j} .
$$

As regard the last $m$ equalities in (13), the proof is the same as the one of Proposition 9: the two lists $L_{m-1}$ and $\bar{L}_{m-1}$ are disjoint and the elements of $\bar{L}_{m-1}$ are pairwise distinct, so one can find a function $\eta \in C^{\infty}\left(X, \mathbf{R}^{d_{y}}\right)$ such that $\eta\left(x_{k}\right)=0$ for $k=0, \ldots, m-1$ while the values of $\eta$ at $\bar{x}_{k}(k=0, \ldots, m-1)$ can be chosen arbitrarily.

Configuration $\mathbf{C}_{4}^{\prime \prime}$. In this case, we have $u_{i}=\ldots u_{p-1}=u_{j}=\ldots u_{m-1}$; the equalities $x_{i}=x_{p}$ and $\bar{x}_{j}=\bar{x}_{m}$ then imply that the trajectories of the vector field $f\left(\cdot, u_{p-1}\right)$ are periodic.

We choose some subscripts $0 \leq i<p \leq 2 n$ and $0 \leq j<m \leq 2 n$, without loss of generality, we assume that $m \geq p$. We consider first t representation $\rho$ defined on $\mathscr{G}_{2}^{U}(a) \times C^{r}(X, \mathbf{R})$ through the mapping $\rho_{f, h}$ defined as

$$
\begin{aligned}
& \rho_{f, h}: \quad X^{(2)} \times U_{(i, p, j, m)}^{m} \times \mathbf{R}_{+}^{*} \quad \longrightarrow \quad X^{m+1} \times X^{m+1} \times \mathbf{R}^{m d_{y}} \\
& \left(x, \bar{x}, \underline{u_{m}}, t\right) \quad \longmapsto\left(x_{0}, \ldots, x_{p-1}, \varphi_{t}^{u_{p_{1}}}\left(x_{i}\right), x_{p+1}, \ldots, x_{m},\right. \\
& \left.\bar{x}_{0}, \ldots, \bar{x}_{m}, y_{0}-\bar{y}_{0}, \ldots, y_{m-1}-\bar{y}_{m-1}\right) \text {. }
\end{aligned}
$$

where $U_{(i, p, j, m)}^{m}$ is the submanifold of $U^{m}$ defined as the set of those $\underline{u_{m}}$ such that $u_{i}=\cdots=u_{p-1}=u_{j}=\cdots=u_{m-1}$.

Together with $\rho$, we consider the submanifold $Z_{j, m}^{i, p}$ defined as in the previous case. We shall prove that $\mathrm{ev}_{\rho}$ is transversal to $Z_{j, m}^{i, p}$. Let $\mathscr{X} \triangleq\left(f, h, x, \bar{x}, u_{m}, t\right)$ be a point such that $\operatorname{ev}_{\rho}(\mathscr{X}) \in Z_{j, m}^{i, p}$ and take a tangent vector $(\mathfrak{X}, \overline{\mathfrak{X}}, \mathfrak{Y})$ with $\mathfrak{X}_{k} \in T_{x_{k}} X$, $k \in\{0, \ldots, m\} \backslash\{p\}, \mathfrak{X}_{p} \in \mathrm{T}_{x_{i}} X, \overline{\mathfrak{X}}_{k}, \in \mathrm{T}_{\bar{x}_{k}} X, k=0, \ldots, m$ and $\mathfrak{Y}_{k} \in \mathbf{R}^{d_{y}}, k=$ $0, \ldots, m-1$. Notice that $\operatorname{ev}_{\rho}(\mathscr{X}) \in Z_{j, m}^{i, p}$ implies that $x_{i}$ and $\bar{x}_{j}$ belong to periodic trajectories of the vector field $f\left(\cdot, u_{p-1}\right)$.

We have to prove that there exist $\phi \in \Gamma_{U}(X), \eta \in C^{r}\left(X, \mathbf{R}^{d_{y}}\right), \xi \in \mathrm{T}_{x} X$, $\bar{\xi} \in \mathrm{T}_{\bar{x}} X, \nu_{k} \in \mathrm{T}_{u_{k}} U, \tau \in \mathbf{R}$, and a vector $\zeta$ in the tangent space to $W_{j, m}^{i, p}$ at $\operatorname{ev}_{\rho}(\mathscr{X})$ 
such that

$$
(\mathfrak{X}, \overline{\mathfrak{X}}, \mathfrak{Y})=\mathrm{d}\left(\operatorname{ev}_{\rho}\right)(\mathscr{X}) \cdot(\phi, \eta, \xi, \bar{\xi}, \nu, \tau)+\zeta .
$$

We shall prove this relation with $\nu=0$ and $\bar{\xi}=0$. We denote by

$$
\alpha_{0}, \ldots, \alpha_{m}, \bar{\alpha}_{0}, \ldots, \bar{\alpha}_{m}, 0, \ldots, 0
$$

the components of $\zeta$; notice that, as $\zeta$ is a tangent vector to $Z_{j, m}^{i, p}$, we have $\alpha_{i}=\alpha_{p}$ and $\bar{\alpha}_{j}=\bar{\alpha}_{m}$.

As in the previous cases, in order to prove that the first $2 m+2$ equations in (15) can be satisfied, it is sufficient to focus our attention to the four following equations

$$
\begin{array}{rlrl}
\alpha_{i}+\xi_{i}+A_{i} & =\mathfrak{X}_{i}, & \alpha_{p}+\xi_{p}+A_{p}+\tau f\left(x_{i}, u_{p-1}\right) & =\mathfrak{X}_{p}, \\
\bar{\alpha}_{j}+\bar{A}_{j} & =\overline{\mathfrak{X}}_{j}, & \bar{\alpha}_{m}+\bar{A}_{m}=\overline{\mathfrak{X}}_{m},
\end{array}
$$

the notations are the same as in the previous cases except for $A_{p}$ and $\xi_{p}$ :

$$
A_{p}=\left.\frac{\mathrm{d} \varphi_{t}^{u_{p-1}, \lambda}\left(x_{i}\right)}{\mathrm{d} \lambda}\right|_{\lambda=0}, \quad \quad \xi_{p}=\mathrm{d} \varphi_{t}^{u_{p-1}}\left(x_{i}\right) \cdot \xi_{i}
$$

We can apply Lemma 8: there exists $\phi$ such that $\bar{A}_{j}=0$ while $\bar{A}_{m}$ can be arbitrarily chosen. Here we cannot ensure that we also have $A_{i}=A_{p}=0$ because $x_{i}$ could belong to the periodic trajectory of $f\left(\cdot, u_{p-1}\right)$ passing through $\bar{x}_{j}$, so we first choose $\bar{\alpha}_{j}=\overline{\mathfrak{X}}_{j}$ and $\bar{A}_{m}=\overline{\mathfrak{X}}_{m}-\overline{\mathfrak{X}}_{j}$. Now as $\varphi_{t}^{u_{p-1}}\left(x_{i}\right)=x_{i}$, we have $t=q \pi_{0}$ where $\pi_{0}$ denotes the prime period of the periodic trajectory of $f\left(\cdot, u_{p-1}\right)$ passing through $x_{i}$; thus $\mathrm{d} \varphi_{t}^{u_{p-1}}\left(x_{i}\right)=\left(\mathrm{d} \varphi_{\pi_{0}}^{u_{p-1}}\left(x_{i}\right)\right)^{q}$. As before, due to the fact that $f$ belongs to $\mathscr{G}_{2}^{U}(a)$, the linear mapping

$$
\left(\xi_{i}, \tau\right) \mapsto\left(\left(\mathrm{d} \varphi_{\pi_{0}}^{v}\left(x_{i}\right)\right)^{q}-\mathrm{Id}\right) \cdot \xi_{i}+\tau f\left(x_{i}, v\right)
$$

is onto. Thus we can find $\xi_{i}$ and $\tau$ such that

$$
\left(\left(\mathrm{d} \varphi_{\pi_{0}}^{v}\left(x_{i}\right)\right)^{q}-\mathrm{Id}\right) \cdot \xi_{i}+\tau f\left(x_{i}, v\right)=\mathfrak{X}_{p}-\mathfrak{X}_{i}+A_{p}-A_{i} .
$$

we take also $\alpha_{i}=\mathfrak{X}_{i}-A_{i}$, with these choices of $\alpha_{i}, \xi_{i}$ and $\tau$, we see that the two first equations are also satisfied.

As regards the last $m$ equations in (15), we argue as in the previous case.

At this point, the application of the Transversal Density Theorem, shows that $\mathscr{R}_{4}$, the set of pairs $(f, h)$ in $\mathscr{G}_{2}^{U}(a) \times C^{r}\left(X, \mathbf{R}^{d_{y}}\right)$ such that $\rho_{f, h}$ is transversal to the finite set of submanifolds $Z_{j, m}^{i, p}$, is a residual. Now, we have to compute the codimension of $Z_{j, m}^{i, p}$, it is equal to $2 n+m d_{y}$ and is greater or equal to $2 n+m d_{u}+m$; this codimension is greater than the dimension of the domain of $\rho_{f, h}$ if $m \geq 2$ or $d_{y} \geq d_{u}+2$. In this case, to be transversal to $Z_{j, m}^{i, p}$ means non membership and we can conclude the proof of Proposition 10 as for the previous propositions. If $m=1, d_{u}=0$ and $d_{y} \geq 1, \mathrm{ev}_{\rho}$ is still transversal to submanifold $Z_{j, m}^{i, p}$ but $\operatorname{codim}\left(Z_{0,1}^{0,1}\right)$ is then equal to the dimension of the domain of $\rho_{f, h}$, so we need an additional argument to conclude in this case. Hereafter, we shall see that, in this particular case, although the codimension of $Z_{0,1}^{0,1}$ is equal to the dimension of the domain of $\rho_{f, h}$, transversality implies non membership. 
Case where $m=1, d_{u}=0$ and $d_{y} \geq 1$. Take $(f, h) \in \mathscr{R}_{4}^{r}$, then $\rho_{f, h}$ is transversal to $W_{0,1}^{0,1}$. Assume that $x_{0} \neq \bar{x}_{0}$ are two points of $X$ such that

- there exists some $t>0$ such that $\varphi_{t}\left(x_{0}\right)=x_{0}, \varphi_{T}^{u}\left(\bar{x}_{0}\right)=\bar{x}_{0}$;

- $h\left(x_{0}\right)=h\left(\bar{x}_{0}\right)$;

then there exist $\xi_{0} \in \mathrm{T}_{x_{0}} X, \bar{\xi}_{0} \in \mathrm{T}_{\bar{x}_{0}} X, \tau \in \mathbf{R}, \alpha_{0}$ and $\bar{\alpha}_{0}$ in the tangent spaces to $X$ at $x_{0}$ and $\bar{x}_{0}$ such that the following equations are satisfied

$$
\left\{\begin{array}{rlrl}
\alpha_{0}+\xi_{0} & =\mathfrak{X}_{0}, & \alpha_{0}+\mathrm{d} \varphi_{t}\left(x_{0}\right) \cdot \xi_{0}+\tau f\left(x_{0}, u\right) & =\mathfrak{X}_{1}, \\
\bar{\alpha}_{0}+\bar{\xi}_{0} & =\overline{\mathfrak{X}}_{0}, & \bar{\alpha}_{0}+\mathrm{d} \varphi_{T}\left(\bar{x}_{0}\right) \cdot \bar{\xi}_{0}=\overline{\mathfrak{X}}_{1} \\
\mathrm{~d} h\left(x_{0}\right) \cdot \xi_{0}-\mathrm{d} h\left(\bar{x}_{0}\right) \cdot \bar{\xi}_{0} & =\mathfrak{Y} & &
\end{array}\right.
$$

whatever $\mathfrak{X}_{0}, \mathfrak{X}_{1}, \overline{\mathfrak{X}}_{0}, \overline{\mathfrak{X}}_{1}$ and $\mathfrak{Y}$ tangent vectors to the appropriate spaces. Clearly the four first equations in this system are equivalent to the two following ones

$$
\begin{aligned}
\left(\mathrm{d} \varphi_{t}\left(x_{0}\right)-\mathrm{Id}\right) \cdot \xi_{0}+\tau f\left(x_{0}, u\right) & =\mathfrak{X}_{1}-\mathfrak{X}_{0}, \\
\left(\mathrm{~d} \varphi_{T}\left(\bar{x}_{0}\right)-\mathrm{Id}\right) \cdot \bar{\xi}_{0} & =\overline{\mathfrak{X}}_{1}-\overline{\mathfrak{X}}_{1} .
\end{aligned}
$$

As 1 is an eigenvalue of the linear mapping $\mathrm{d} \varphi_{T}\left(\bar{x}_{0}\right)$, clearly the second equation of this system cannot be satisfied. This implies that if $\varphi_{T}\left(x_{0}\right)=x_{0}$ and $\varphi_{T}\left(\bar{x}_{0}\right)=\bar{x}_{0}$, the point $\rho_{f, h}\left(x_{0}, \bar{x}_{0}\right)$ cannot belong to $W_{0,1}^{0,1}$, which means that we must have $h\left(x_{0}\right) \neq$ $h\left(\bar{x}_{0}\right)$. This achieve the proof of Proposition 10.

5. The case $d_{u} \geq 1$ and $d_{y}=d_{u}+1$. Counterexample. We shall exhibit here a simple counterexample which shows that if $d_{y}=2$ and $d_{u}=1$, then the conclusion of our main result (Theorem 4 ) is no more true. That it to say, we exhibit a pair $\left(f_{0}, h_{0}\right)$ such that for every $(f, h)$ in some neighborhood of $\left(f_{0}, h_{0}\right)$, the related mapping $\Theta_{T}^{f, h}$ is not injective. We recall hereafter, the isotopy theorem [1] which will be used to prove some optimality of our main result.

Theorem 11 (Transversal isotopy theorem). Let $\mathscr{A}, Z$ and $Y$ be $C^{r+1}$ manifolds $(r \geq 1), \rho: \mathscr{A} \rightarrow C^{r+1}(Z, Y)$ a $C^{r+1}$ representation, $W \subset Y$ a submanifold and $a_{0} \in \mathscr{A}$ a point. For $a \in \mathscr{A}$ let $W_{a}=\rho_{a}^{-1}(W)$. Assume that

1. $W$ is closed in $Y$;

2. $Z$ is compact and $C^{r+3}$;

3. $\rho_{a_{0}}$ is transversal to $W$.

Then there is an open neighborhood $N$ of $a_{0}$ in $\mathscr{A}$ such that, for $a \in N$, there is a $C^{r}$ diffeomorphism $F_{a}: Z \rightarrow Z$ such that $F_{a}\left(W_{a_{0}}\right)=W_{a}$ and $F_{a}$ is $C^{r}$ isotopic to the identity.

We shall apply the transversal isotopy theorem 11 to the following situation: we take

- $\mathscr{A}=\Gamma_{U}(X) \times C^{\infty}\left(X, \mathbf{R}^{d_{y}}\right)$, where $X$ and $U$ are compact manifolds, $\operatorname{dim} U=$ $d_{u}>0$ with $d_{y}=d_{u}+1$

- $Z=X^{2} \times U \times S^{1}$;

- $Y=X^{3} \times \mathbf{R}^{d_{y}}$.

We consider also a representation $\rho$ which is slightly different from the one which has been used in the proof of Proposition 10; we define this representation through $\rho_{f, h}$ as

$$
\begin{aligned}
\rho_{f, h}: \quad X^{2} \times U \times S^{1} & \longrightarrow X^{3} \times \mathbf{R}^{d_{y}} \\
(x, \bar{x}, u, s) & \longmapsto\left(x, \varphi_{T}^{u}(x), \varphi_{\gamma(s)}^{u}(\bar{x}), h(x)-h(\bar{x})\right)
\end{aligned}
$$


here $\gamma \in C^{\infty}\left(S^{1}, \mathbf{R}\right)$.

The submanifold $W \subset X^{3} \times \mathbf{R}^{d_{y}}$ is then defined as

$$
W=\left\{\left(z_{1}, z_{2}, z_{3}, 0\right) \in X^{3} \times \mathbf{R}^{d_{y}} \mid z_{1}=z_{2}=z_{3}\right\} .
$$

Consider a pair $\left(f_{0}, h_{0}\right)$ such that $\rho_{f_{0}, h_{0}}$ is transversal to $W$ and such that $W_{f_{0}, h_{0}}$ is nonempty. Applying the Transversal isotopy theorem 11, we deduce that there exists a neighborhood $N$ of $\left(f_{0}, h_{0}\right)$ such that if $(f, h) \in N, W_{f, h}=F\left(W_{f_{0}, h_{0}}\right)$ with $F$ a diffeomorphism from $Z$ to $Z$. Thus $W_{f, h}$ is nonempty; notice that we can assume that $\rho_{f, h}$ is transversal to $W$ for every pair $(f, h) \in N$, this is a direct consequence of the Openness of Transversal Intersection Theorem [1] applied to this situation with $K=Z$. Let $(x, \bar{x}, u, s)$ be an element of $W_{f, h}$, we shall show that $x \neq \bar{x}$ : arguing by contradiction, we shall see that if we have the equality $x=\bar{x}$, then $\rho_{f, h}$ cannot be transversal to $W$ at $(x, \bar{x}, u, s)$. We introduce some notations

$$
\begin{array}{ll}
A=\mathrm{d} \varphi_{T}^{u}(x), & \bar{A}=\mathrm{d} \varphi_{\gamma(s)}^{u}(\bar{x}), \\
B=\left.\frac{\partial \varphi_{T}^{v}(x)}{\partial v}\right|_{v=u}, & \bar{B}=\left.\frac{\partial \varphi_{\gamma(s)}^{v}(\bar{x})}{\partial v}\right|_{v=u}, \\
C=\mathrm{d} h(x), & \bar{C}=\mathrm{d} h(\bar{x}) .
\end{array}
$$

Consider now the following "matrix" $M$

$$
M=\left(\begin{array}{cccc}
A-\mathrm{Id} & 0 & B & 0 \\
-\mathrm{Id} & \bar{A} & \bar{B} & f(x, u) \\
C & -\bar{C} & 0 & 0
\end{array}\right) .
$$

Arguing as in the proof of Proposition 10, we see that the transversality of $\rho_{f, h}$ to $W$ at $(x, \bar{x}, u, s)$ is equivalent to the invertibility of the square matrix $M$. Since we assume that $x=\bar{x}$, we have $C=\bar{C}$, which implies that the determinant of $M$ is equal to the one of the following matrix $M^{\prime}$ :

$$
M^{\prime}=\left(\begin{array}{cccc}
A-\mathrm{Id} & 0 & B & 0 \\
\bar{A}-\mathrm{Id} & \bar{A} & \bar{B} & f(x, u) \\
0 & -\bar{C} & 0 & 0
\end{array}\right) .
$$

Now as $f(x, u)$ belongs to the kernels of $A-\operatorname{Id}$ and $\bar{A}-\operatorname{Id}$ (because $\varphi_{\gamma(t)}^{u}(\bar{x})=x$ ), the vector $(f(x, u), 0,0,0)^{\mathrm{T}}$ belongs to the kernel of $M^{\prime}$ which implies that the determinant of $M^{\prime}$ is zero : we have reached a contradiction. As a consequence, if $(f, h) \in N$, there exists $x \neq \bar{x}, u$ and $s$ such that $h(x)=h(\bar{x})$; moreover, the trajectory of $f(\cdot, u)$ passing through $x$ is periodic and $\bar{x}$ belongs to this trajectory, so we have $\varphi_{k T}^{u}(x)=x$ and $\varphi_{k T}^{u}(\bar{x})=\bar{x}$.

We give now an explicit example of a pair $\left(f_{0}, h_{0}\right)$ such that $\rho_{f_{0}, h_{0}}$ is transversal to $W$. In what follows, for the sake of simplicity and without loss of generality, we assume that $T=2 \pi$. The manifold $X$ will be equal to the circle $S^{1}$ and the set of controls $U$ will also be equal to the circle $S^{1}$. We denote by $u_{1}$ and $u_{2}$ (resp. $s_{1}$ and $s_{2}$ ) the components of $u$ (resp. of $s$ ) and we consider the following vector field:

$$
f_{0}(x, u)=R_{1} \cdot x+u_{1} R_{2} \cdot x
$$


with $R_{1}$ and $R_{2}$ the following skew-symmetric matrices

$$
R_{1}=\left(\begin{array}{cc}
0 & -1 \\
1 & 0
\end{array}\right), \quad R_{2}=\left(\begin{array}{cc}
0 & -\beta \\
\beta & 0
\end{array}\right),
$$

with $\beta \in(0,1)$.

Mapping $h_{0}$ is defined by:

$$
\begin{aligned}
h_{0}: X & \longrightarrow \mathbf{R}^{2} \\
x & \longmapsto\left(x_{1}, x_{1} x_{2}\right),
\end{aligned}
$$

while function $\gamma$ is defined by:

$$
\gamma(s)=\left(-\frac{1}{2}+\frac{1}{4} s_{1}\right) 2 \pi .
$$

The set $\boldsymbol{W}_{f_{0}, h_{0}}$. Let $x=\left(x_{1}, x_{2}\right)$ and $\bar{x}=\left(\bar{x}_{1}, \bar{x}_{2}\right)$ be two points in $S^{1}$, and $u=\left(u_{1}, u_{2}\right) \in S^{1}$ a control and $s \in S^{1}$; assume that $\rho_{f_{0}, h_{0}}(x, \bar{x}, u, s) \in W$. Letting $z=x_{1}+i x_{2} \in \mathbf{C}$, the equality $\varphi_{T}^{u}(x)=x$ is equivalent to $e^{i\left(1+u_{1} \beta\right) 2 \pi} z=z$ which is equivalent to $1+u_{1} \beta=k$ with $k \in \mathbf{Z}$. Now, as $\beta \in(0,1)$, we have $\left|u_{1} \beta\right|<1$ and so the equality $u_{1} \beta=k-1 \in \mathbf{Z}$ is possible if and only if $u_{1}=0$. From the definition of $h$, we can easily see that the equality $h(x)=h(\bar{x})$ amounts to $x=\bar{x}$ or $x_{1}=\bar{x}_{1}=0$. If $x=\bar{x}$, as $u_{1}=0$, the equality $\varphi_{\gamma(s)}^{u}(\bar{x})=x$ is possible only if $\gamma(s) \in \mathbf{Z}$, but from the definition of $\gamma$, we have $\gamma(s) \in[-3 / 4,-1 / 4]$. Thus $x \neq \bar{x}$ and $x_{1}=\bar{x}_{1}=0$, so, taking into account that $u_{1}=0$, the equality $\varphi_{\gamma(s)}^{u}(\bar{x})=x$ is equivalent to the following ones

$$
-\sin (\gamma(s)) \bar{x}_{2}=0, \quad \cos (\gamma(s)) \bar{x}_{2}=x_{2}=-\bar{x}_{2} .
$$

These two equalities are true iff $\gamma(s)=(2 k+1) \pi$ with $k \in \mathbf{Z}$, which is equivalent to $s_{1}=4 k+4$, as $\left|s_{1}\right| \leq 1$, this is possible only if $s_{1}=0$.

In conclusion, $W_{f_{0}, h_{0}}$ is the set consisting of the following eight elements

$$
W_{f_{0}, h_{0}}=\left\{\left(\left(0, \varepsilon_{0}\right),\left(0,-\varepsilon_{0}\right),\left(0, \varepsilon_{1}\right),\left(0, \varepsilon_{2}\right)\right)\right\} .
$$

where $\varepsilon_{0}, \varepsilon_{1}, \varepsilon_{2} \in\{-1,1\}$.

The transversality of $\boldsymbol{\rho}_{f_{0}, h_{0}}$. Take $(x, \bar{x}, u, s) \in X \times X \times U \times S^{1}$ an element such that $\rho_{f_{0}, h_{0}}(x, \bar{x}, u, s)$ belongs to $W$. Thus we have

$$
x=\left(0, \varepsilon_{0}\right), \quad \bar{x}=\left(0,-\varepsilon_{0}\right), \quad u=\left(0, \varepsilon_{1}\right), \quad s=\left(0, \varepsilon_{2}\right)
$$

with $\varepsilon_{i} \in\{-1,1\}(i=0,1,2)$. Let $\left(\mathfrak{X}_{1}, \mathfrak{X}_{2}, \mathfrak{X}_{3}, \mathfrak{Y}\right)$ be a tangent vector to $X^{3} \times \mathbf{R}^{2}$ at $\rho_{f_{0}, h_{0}}(x, \bar{x}, u, s)$. Thus, $\mathfrak{X}_{1}, \mathfrak{X}_{2}$ and $\mathfrak{X}_{3}$ are tangent to $S^{1}$ at $\left(0, \varepsilon_{0}\right)$ while $\mathfrak{Y}$ is a vector in $\mathbf{R}^{2}$. We write a tangent vector to submanifold $W$ as $\zeta=(\alpha, \alpha, \alpha, 0)$ where $\alpha$ is a tangent vector to $S^{1}$ at $\left(0, \varepsilon_{0}\right)$. We have to prove that there exists $\xi$ (resp. $\bar{\xi}$ ) in the tangent space to $S^{1}$ at $\left(0, \varepsilon_{0}\right)$ (resp. $\left.\left(0,-\varepsilon_{0}\right)\right)$ as well as $\nu$ a vector in the tangent space to $S^{1}$ at $\left(0, \varepsilon_{1}\right)$ and $\sigma$ a vector tangent to $S^{1}$ at $s$ such that the following equalities are satisfied

$$
\begin{aligned}
& \mathfrak{X}_{1}=\xi+\alpha, \\
& \mathfrak{X}_{2}=\mathrm{d} \varphi_{T}^{u}(x) \cdot \xi+\frac{\partial \varphi_{T}^{u}(x)}{\partial u} \cdot \nu+\alpha, \\
& \mathfrak{X}_{3}=\mathrm{d} \varphi_{\gamma(s)}^{u}(\bar{x}) \cdot \bar{\xi}+\frac{\partial \varphi_{\gamma(s)}^{u}(\bar{x})}{\partial u} \cdot \nu+\frac{\partial \varphi_{\gamma(s)}}{\partial s} \cdot \sigma+\alpha, \\
& \mathfrak{Y}=\mathrm{d} h(x) \cdot \xi-\mathrm{d} h(\bar{x}) \cdot \bar{\xi} .
\end{aligned}
$$


We examine first the fourth equation (21), taking into account that $\xi=\left(\xi_{1}, 0\right)^{\mathrm{T}}$ and $\bar{\xi}=\left(\bar{\xi}_{1}, 0\right)^{\mathrm{T}}$, it writes

$$
\left(\begin{array}{ll}
1 & 0 \\
\varepsilon_{0} & 0
\end{array}\right)\left(\begin{array}{c}
\xi_{1} \\
0
\end{array}\right)-\left(\begin{array}{cc}
1 & 0 \\
-\varepsilon_{0} & 0
\end{array}\right)\left(\begin{array}{c}
\bar{\xi}_{1} \\
0
\end{array}\right)=\left(\begin{array}{l}
\mathfrak{Y}_{1} \\
\mathfrak{Y}_{2}
\end{array}\right)
$$

Clearly, the solution of this equation is

$$
\xi_{1}=\frac{\varepsilon_{0} \mathfrak{Y}_{2}+\mathfrak{Y}_{1}}{2}, \quad \bar{\xi}_{1}=\frac{\varepsilon_{0} \mathfrak{Y}_{2}-\mathfrak{Y}_{1}}{2} .
$$

Taking into account that $\mathfrak{X}_{i}=\left(\mathfrak{X}_{i}^{1}, 0\right)^{\mathrm{T}}$ and $\alpha=\left(\alpha_{1}, 0\right)^{\mathrm{T}}$, the solution of the first equation (18) is then given by (22) and by the equality

$$
\alpha_{1}=\mathfrak{X}_{1}^{1}-\frac{\varepsilon_{0} \mathfrak{Y}_{2}+\mathfrak{Y}_{1}}{2} .
$$

Concerning the second equation (19), we notice that $\mathrm{d} \varphi_{T}^{u}(x)=\mathrm{Id}$ and that

$$
\frac{\partial \varphi_{T}^{u}(x)}{\partial u}=\left(\begin{array}{cc}
-2 \pi \beta \varepsilon_{0} & 0 \\
0 & 0
\end{array}\right)
$$

so the solution of equation (19) is given by (22), (23) and by

$$
\nu_{1}=-\frac{\mathfrak{X}_{2}^{1}-\mathfrak{X}_{1}^{1}}{2 \pi \beta \varepsilon_{0}} .
$$

So far, the values of $\xi, \bar{\xi}$ and $\nu$ are fixed, therefore showing that equation (20) comes down showing that the third term in the right-hand member of (20) can take arbitrary value. This third term writes

$$
\frac{\partial \varphi_{\gamma(s)}}{\partial s} \cdot \sigma=\frac{\pi}{2} \sigma_{1} f\left(\varphi_{\gamma(s)}^{u}(x)\right)=\frac{\pi}{2} \sigma_{1}\left(\begin{array}{c}
\varepsilon_{0} \\
0
\end{array}\right),
$$

obviously, thanks to a suitable choice of $\sigma_{1}$, this expression can be made equal to an arbitrary tangent vector $\tilde{\mathfrak{X}}_{3}$ of $S^{1}$ at $x$.

This achieves the proof of the transversality of $\rho_{f_{0}, h_{0}}$ to $W$.

Appendix A. Proof of Theorem 7. The proof of the Kupka-Smale theorem can obviously be found in the original papers $[15,16]$ and $[17]$ but the reader can also find a very detailed proof of this result in [1]. For the proof of the generalization of this theorem, we shall follow closely the arguments given in this book.

\section{A.1. The different sets in the theorem of Kupka-Smale.}

G1 Set $\mathscr{G}_{1}$ is the set of vector fields whose critical points are elementary. A point $x$ is critical for the vector field $f$ if $f(x)=0$, it is elementary if the differential

$$
\mathrm{d} \varphi_{t}(x): \mathrm{T}_{x} X \longrightarrow \mathrm{T}_{x} X
$$

has no complex eigenvalue of modulus 1 for every $t \neq 0$.

G $\Delta$ Let $a$ be a positive number, set $\mathscr{G} \Delta(a)$ is the set of vector fields $f$ such that if $\mathcal{O}$ is a closed orbit of $f$ with period $0<\tau \leq a$, then this period is transversal, that is to say, the eigenvalue 1 of the differential

$$
\mathrm{d} \varphi_{\tau}(x): \mathrm{T}_{x} X \longrightarrow \mathrm{T}_{x} X
$$

has an algebraic multiplicity equal to 1 (notice that, in this case, $f(x)$ is an eigenvector of $\left.\mathrm{d} \varphi_{\tau}(x)\right)$. 
G3/2 Set $\mathscr{G}_{3 / 2}(a)$ is defined as $\mathscr{G}_{3 / 2}(a) \triangleq \mathscr{G}_{1} \cap \mathscr{G} \Delta(a)$.

G2 Set $\mathscr{G}_{2}(a)$ is the set of vector fields included in $\mathscr{G}_{1}$ and whose periods $0<\tau \leq a$ of closed orbits are elementary. A period is elementary if the modulus of eigenvalues of $\mathrm{d} \varphi_{\tau}(x)$ that are distinct from 1 , are different from 1 .

A consequence of the Kupka-Smale theorem is that all of these sets are open and dense for the $C^{r}$ topology in $\Gamma(X)$.

We recall that $\Gamma_{U}(X)$ denotes the set of parametrized vector fields over $X$; we define the sets $\mathscr{G}_{1}^{U}, \mathscr{G}_{\Delta}^{U}, \ldots$ as the sets of vector fields belonging to one of the above categories for every $u \in U$. For instance we define $\mathscr{G}_{1}^{U}$ as the set of parametrized vector fields $f$ such that $f(\cdot, u) \in \mathscr{G}_{1}$ for every $u \in U$. Notice that every vector field $f \in \Gamma(X)$ can be regarded as a parametrized vector field, so we can write $\Gamma(X) \subset \Gamma_{U}(X)$, $\mathscr{G}_{1} \subset \mathscr{G}_{1}^{U}$, etc.

The tangent bundle $\mathrm{T}(X \times U)$ is diffeomorphic to the cartesian product $\mathrm{T} X \times \mathrm{T} U$, so a vector field $f$ defined on $X \times U$ can be regarded as a pair $\left(f_{1}, f_{2}\right)$ of two vector fields defined respectively on $X$ and $U$. We have then the following result.

Lemma 12. The mapping $\Pi$ defined as

$$
\begin{aligned}
\Pi: \Gamma(X \times U) & \longrightarrow \Gamma_{U}(X) \\
\left(f_{1}, f_{2}\right) & \longmapsto f_{1}
\end{aligned}
$$

is open for the $C^{r}$ topology (for any $r>0$ ).

Proof. The set $C^{\infty}(X \times U, \mathrm{~T} X \times \mathrm{T} U)$ is diffeomorphic to $C^{\infty}(X \times U, \mathrm{~T} X) \times$ $C^{\infty}(X \times U, \mathrm{~T} U)$ (see [12, Prop. 3.6]); we deduce easily that $\Gamma(X \times U)$ is diffeomorphic to $\Gamma_{U}(X) \times \Gamma_{X}(U)$. On the other hand the first projection map from $\Gamma_{U}(X) \times \Gamma_{X}(U)$ to $\Gamma_{U}(X)$ is an open and continuous mapping, it follows that $\Pi$ is continuous and open as a composite mapping of an open and continuous mapping with a diffeomorphism. $\square$

As a consequence of this lemma, the direct image of an open and dense subset of $\Gamma(X \times U)$ is an open and dense subset of $\Gamma_{U}(X)$.

We recall also that a mapping $f: E \rightarrow F$ between topological spaces is called quasi-open if, for every open subset $\mathcal{O} \subset E, f(\mathcal{O})$ has a non empty interior.

We shall need also, the following lemma.

Lemma 13. The mapping $\Phi$ defined as

$$
\begin{aligned}
\Phi^{:} \quad \Gamma_{U}(X) \times U & \longrightarrow \Gamma(X) \\
(f, u) & \longmapsto f_{u}
\end{aligned}
$$

is continuous. Here $f_{u}$ denotes the vector field defined on $X$ by $f_{u}(x)=f(x, u)$.

A.2. The set $\mathscr{G}_{1}^{U}$ is open and dense in $\Gamma_{U}(X)$. The proof of the KupkaSmale theorem 6 as made in [1] needs seven steps. Beforehand, one proves that $\mathscr{G}_{1}^{U}$ is open and dense for the $C^{r}$ topology. The proof follows closely the one provided in [1, page $98 \mathrm{ff}]$, in this book, the authors introduce a submanifold $W$ of the 1-jet bundles $J^{1}(X, \mathrm{~T} X)$ as follows. Consider a chart $(P, \varphi)$ of $X$, to this chart we relate, as usual, the chart $(Q, \psi)$ of $\mathrm{T} X$, where $Q=\pi^{-1}(P)$ (here $\pi: \mathrm{T} X \rightarrow X$ denotes the canonical projection) and $\psi(v)=(\pi(v), \bar{v})$ with $\bar{v}$ the local expression of $v \in \mathrm{T} X$ in the chart $(P, \varphi)$. Define then the chart $\tau_{P, Q}$ as

$$
\begin{aligned}
\tau_{P, Q}: \quad J^{1}(P, Q) & \longrightarrow P^{\prime} \times Q^{\prime} \times \mathcal{L}\left(\mathbf{R}^{n}, \mathbf{R}^{n}\right) \\
\sigma & \longmapsto\left(x, \psi \circ f \circ \varphi^{-1}(x), A\right)
\end{aligned}
$$

where $P^{\prime}=\varphi(P), Q^{\prime}=\psi(Q)$ and $x$ is the source of the 1-jet $\sigma$. The submanifold $W$ is defined as the set of 1 -jets such that $\psi \circ f \circ \varphi^{-1}(x)=0$ and $A$ has at least 
one eigenvalue with real part zero (clearly, this definition does not depend on the particular choice of charts). To $W$, one relates the subset $M$ of $\mathcal{L}\left(\mathbf{R}^{n}, \mathbf{R}^{n}\right)$ of linear maps having an eigenvalue with real part zero. In [1], the proof of the density of $\mathscr{G}^{1}(X)$ relies on the fact that $M$ is closed and is a finite union of submanifolds of codimension $\geq 1$, which implies that $\operatorname{codim} W \geq n+1$. In our case, we consider the same 1-jet bundle but we replace $X$ by $X \times U$, so we can see $A$ as a linear map from $\mathbf{R}^{n+d_{u}}$ to $\mathbf{R}^{n+d_{u}}$, we can identify $A$ with its matrix written in the canonical basis of $R^{n+d_{u}}$ and we write $A$ as a block matrix $A=\left(\begin{array}{ll}A_{11} & A_{12} \\ A_{21} & A_{22}\end{array}\right)$ with $A_{11} \in \mathbf{R}^{n \times n}$ and $A_{22} \in \mathbf{R}^{d_{y}} \times \mathbf{R}^{d_{y}}$. We modify the definition of $W$ as follows: $W$ is the set of 1-jets such that $A_{11}$ has at least one eigenvalue with real part zero. In a similar way as above, we can prove that $W$ has a codimension $\geq n+d_{u}+1$. Consider now the set $\mathscr{G}_{1}^{\prime}(X \times U)$ of vector fields $f$ defined on $X \times U$ such that the 1 -jet of $f$ does not belong to $W$; arguing as in [1], the fact that $\operatorname{codim} W \geq n+d_{u}+1$, proves that the set is open and dense. As $\Pi$ is an open mapping (cf Lemma 12), and as $\Pi\left(\mathscr{G}_{1}^{\prime}(X \times U)\right)=\mathscr{G}_{1}^{U}$, we have proved that $\mathscr{G}_{1}^{U}$ is also open and dense.

\section{A.3. The seven steps of the proof.}

Step 1: for every $a>0$, the set $\mathscr{G}_{2}^{U}(a)$ is open. First notice that $\mathscr{G}_{2}^{U}(a) \neq \varnothing$ because $\mathscr{G}_{2}(a) \subset \mathscr{G}_{2}^{U}(a)$; moreover, we shall use the fact that $\mathscr{G}_{2}(a)$ is open (proven in [1]).

Let $f \in \mathscr{G}_{2}^{U}(a)$ and $u \in U$ be fixed, denote by $f_{u}$ the vector fields in $\Gamma(X)$ defined by $f_{u}(x)=f(x, u) ; f_{u}$ belongs to $\mathscr{G}_{2}(a)$, hence there exists $\mathscr{N}_{u}$ a neighborhood of $f_{u}$ in $\Gamma(X)$ with $\mathscr{N}_{u} \subset \mathscr{G}_{2}(a)$. As the mapping $\Phi$ is continuous ( $c f$ Lemma 13), there exists $\mathscr{N}_{u}^{U}$ a neighborhood of $f$ in $\Gamma_{U}(X)$ and $\mathscr{V}_{u}$ neighborhood of $u$ such that $\Phi(g, v) \in \mathscr{N}_{u}$ for every $(g, v) \in \mathscr{N}_{u}^{U} \times \mathscr{V}_{u}$. The neighborhoods $\mathscr{V}_{u}$ cover $U$, as $U$ is compact, there exists a finite subcover: $U \subset \bigcup_{i=1}^{N} \mathscr{V}_{u_{i}}$, let $\mathscr{N}^{U}=\bigcap_{i=1}^{N} \mathscr{N}_{u_{i}}^{U}, \mathscr{N}^{U}$ is a neighborhood of $f$ in $\Gamma_{U}(X)$. Let $(g, v) \in \mathscr{N}^{U} \times U$, there exists $1 \leq i \leq N$ such that $v \in \mathscr{V}_{u_{i}}$, on the other hand $g \in \mathscr{N}^{U} \subset \mathscr{N}_{u_{i}}^{U}$, therefore $\Phi(g, v)=g_{v}$ belongs to $\mathscr{G}_{2}(a)$.

In conclusion, every element $g$ of $\mathscr{N}^{U}$ is such that $g_{v} \in \mathscr{G}_{2}(a)$ for every $v$ in $U$, therefore $f \in \mathscr{N}^{U} \subset \mathscr{G}_{2}^{U}(a)$, we have showed that $\mathscr{G}_{2}^{U}(a)$ is open.

Step 2: for every $a>0$, the set $\mathscr{G}_{3 / 2}^{U}$ is open. The proof is very similar to the previous one.

Step 3: if $f \in \mathscr{G}_{1}^{U}$, there exists a neighborhood $\mathscr{N}$ of $f$ with $\mathscr{N} \subset \mathscr{G}_{1}^{U}$ and there exists $a_{0}>0$ such that $\mathscr{G}_{2}^{U}\left(a_{0}\right) \cap \mathscr{N}=\mathscr{N}$. . Let $f \in \mathscr{G}_{1}^{U}$, for every $u \in U, f_{u} \in \mathscr{G}_{1}$, so there exists a neighborhood $\mathscr{N}_{u} \subset \mathscr{G}_{1}$ of $f_{u}$ in $\mathscr{G}_{2}(a)\left(f_{u} \in \mathscr{N}_{u} \subset \mathscr{G}_{1}\right)$ and there exists $a_{u}>0$ such that $\mathscr{N}_{u} \subset \mathscr{G}_{2}\left(a_{u}\right)$. As in step 1 , as $\Phi$ is continuous, we construct a family of neighborhoods $\mathscr{N}_{u}^{U}$ of $f$ in $\Gamma_{U}(X)$ and a family $\mathscr{V}_{u}$ of neighborhoods of $u$ such that for every $(g, v) \in \mathscr{N}_{u}^{U} \times \mathscr{V}_{u}$, the vector field $g_{v}$ belongs to $\mathscr{N}_{u} \subset \mathscr{G}_{1}$ and $\mathscr{N}_{u} \subset \mathscr{G}_{2}\left(a_{u}\right)$. Notice that, since $\mathscr{G}_{1}^{U}$ is open, we can assume that $\mathscr{N}_{u}^{U} \subset \mathscr{G}_{1}^{U}$.

Due to the compactness of $U$, we have $U \subset \bigcup_{i=1}^{N} \mathscr{V}_{u_{i}}$; let

$$
\mathscr{N}=\bigcap_{i=1}^{N} \mathscr{N}_{u_{i}}^{U}, \quad \quad a_{0}=\min _{1 \leq i \leq N} a_{u_{i}} .
$$

Let $(g, v) \in \mathscr{N}^{U} \times U$, there exists $1 \leq i \leq N$ such that $v \in \mathscr{V}_{u_{i}}$ and we have $g \in \mathscr{N} \subset$ $\mathscr{N}_{u_{i}}^{U}$, therefore $\Phi(g, v)=g_{v} \in \mathscr{N}_{u_{i}}$ and $\mathscr{N}_{u_{i}} \subset \mathscr{G}_{2}\left(a_{u_{i}}\right)$, but, clearly, $\mathscr{G}_{2}\left(a_{u_{i}}\right) \subset \mathscr{G}_{2}\left(a_{0}\right)$ since $a_{0} \leq a_{u_{i}}$. Thus, we have showed that

$$
f \in \mathscr{N} \subset \mathscr{G}_{1}^{U} \cap \mathscr{G}_{2}^{U}\left(a_{0}\right)
$$

which achieves the proof. 
Step 4: if $\mathscr{N} \subset \mathscr{G}_{1}^{U}$ is open and $\mathscr{G}_{2}^{U}(a) \cap \mathscr{N}$ is dense in $\mathscr{N}$ then $\mathscr{G}_{3 / 2}^{U}\left(\frac{3}{2} a\right) \cap \mathscr{N}$ is dense in $\mathscr{N}$.. This step is based on lemmas 31.7 and 31.8 in [1, p. 102]. Lemma 30.7 asserts that if $x$ is a point located on a closed orbit of a vector field $f \in \Gamma(X)$, given a tangent vector $v \in \mathrm{T}_{x} X$, there exists a vector field $g \in \Gamma(X)$ such that, denoting by $\tau$ the prime period of the closed trajectory and by $\varphi_{t}^{\lambda}$ the flow related to $f+\lambda g$, we have

$$
\left.\frac{\mathrm{d} \varphi_{\tau}^{\lambda}(x)}{\mathrm{d} \lambda}\right|_{\lambda=0}=v .
$$

As pointed out in section 4.2, Lemma 31.7 is still true for parametrized vector fields. Moreover, Lemma 31.8 can be proved in exactly the same way for parametrized vector fields, the representation $\rho$ being modified as follows (we define it through the evaluation map):

$$
\begin{array}{cl}
\mathrm{ev}_{\rho}: \quad \Gamma_{U}(X) \times U \times X \times \mathbf{R}_{+}^{*} & \longrightarrow X \times \mathbf{R}_{+}^{*} \times X \\
(f, u, x, t) & \longmapsto\left(x, t, \varphi_{t}^{u}(x)\right)
\end{array}
$$

Finally, we can prove that $\mathrm{ev}_{\rho}$ is transverse to $\Delta$ on $\mathscr{G}_{2}^{U}(a) \times X \times U \times\left[0, \frac{3}{2}+\varepsilon\right)$ in the same way as for the proof written in [1] because $X \times U$ is compact; we can then conclude exactly as in [1].

Step 5: if $\mathscr{N} \subset \mathscr{G}_{1}^{U}$ is open and if $\mathscr{G}_{3 / 2}^{U}(a) \cap \mathscr{N}$ is dense in $\mathscr{N}$ then $\mathscr{G}_{2}^{U}(a) \cap \mathscr{N}$ is dense in $\mathscr{N}$.. In [1], the proof of this step is needs four lemmas. We shall briefly indicate how they can be extended to the case of parametrized vector fields. In the sequel, we shall identify the tangent bundle $\mathrm{T}(X \times U)$ with the product $\mathrm{T} X \times \mathrm{T} U$. Take a parametrized vector field, $f \in \Gamma_{U}(X)$ and consider the related vector field $\tilde{f}$ defined on $X \times U$ as $\tilde{f}_{1}(x, u)=f(x, u)$ and $\tilde{f}_{2}(x, u)=0$. Assume that $x \in X$ belongs to a periodic trajectory, denoted by $\gamma$, of $f(\cdot, u)$, then $(x, u)$ belongs to the periodic trajectory $\tilde{\gamma} \triangleq \gamma \times\{u\}$ of $\tilde{f}$. We can apply the tangent perturbation lemma (Lemma 32.4 in [1]), to $\tilde{f}$ and $\tilde{\gamma}$ : we then obtain a vector field $\tilde{g}$ defined on $X \times U$ that is zero on $\tilde{\gamma}$ and zero outside some neighborhood of $\tilde{\gamma}$. Moreover if we denote by $\tilde{\varphi}^{\lambda}$ the flow of $\tilde{f}+\lambda \tilde{g}$, we have $\left.\frac{\mathrm{d}}{\mathrm{d} \lambda}\left\{d \varphi_{\tau}(x, u)\right\}\right|_{\lambda=0}=\tilde{A}$ where $\tilde{A}$ is endomorphism of $T_{x} X \times \mathrm{T}_{u} U$ that vanishes at $(f(x, u), 0)^{\mathrm{T}}$. In particular, if we choose $\tilde{A}$ such that its second component is zero, then looking at the proof of Lemma 32.4, we see that, $\tilde{g}_{2}$, the second component of $\tilde{g}$, is zero. This lemma is therefore still valid for parametrized vector fields.

Consider now a parametrized vector field $f \in \mathscr{G}_{3 / 2}^{U}(a)$ and assume that $x_{0}$ be a point on a periodic trajectory $\gamma_{0}$ of $f\left(\cdot, u_{0}\right)$, if $u$ is closed to $u_{0}$, then the vector field $f(\cdot, u)$ is closed to $f\left(\cdot, u_{0}\right)$. Invoking Lemma 24.4 in $[1]$, we know that there exists some neighborhood of $x_{0}$ such that the vector field $f(\cdot, u)$ admits a unique periodic trajectory in this neighborhood if $u$ is closed enough to $u_{0}$. We can be more specific by using the implicit function theorem. Take a submanifold $Y \subset X$ of codimension 1 , passing through $x_{0}$ and which intersects $\gamma_{0}$ transversally at $x_{0}$. We consider the Poincare map $\mathcal{P}$, related to the vector field $f\left(\cdot, u_{0}\right)$, defined in a neighborhood of $x_{0}$ in $Y$. This map is also defined for $u$ closed enough to $u_{0}$, that is to say, there exists neighborhoods $N_{1} \subset Y$ of $x_{0}$ in $Y$ and $N_{2}$ of $u_{0}$ such that if $(x, u) \in N_{1} \times N_{2}$, there exists a first time $\tau_{u}>0$ such that $\varphi_{\tau_{u}}^{u}(x, u) \in N_{1}$. Now, $\mathcal{P}\left(x_{0}, u_{0}\right)=x_{0}$ and we can invoke the implicit function theorem to prove the existence of $x_{u}$ and $\tau_{u}$ defined for $u$ in some neighborhood of $u_{0}$ included in $N_{2}$ and such that $\mathcal{P}\left(x_{u}, u\right)=x_{u}$ (as well as $\left.\varphi_{\tau_{u}}^{u}\left(x_{u}\right)=x_{u}\right)$. To see why we can use this theorem, we use the assumption that $f \in \mathscr{G}_{3 / 2}^{U}(a)$. We know that 1 is an eigenvalue of $\mathrm{d} \varphi_{\tau_{u_{0}}}^{u_{0}}\left(x_{0}\right)$ with multiplicity one and 
that the other eigenvalues of $\mathrm{d} \varphi_{\tau_{u_{0}}}^{u_{0}}\left(x_{0}\right)$ are different from 1 . The vector space $T_{x_{0}} X$ can then be written as $T_{x_{0}} X=\mathbf{R} f\left(x_{0}, u_{0}\right) \oplus E$ where $E$ is the $n-1$ dimensional subspace of $\mathrm{T}_{x_{0}} X$ generated by the eigenvectors of $\mathrm{d} \varphi_{\tau_{u_{0}}}^{u_{0}}\left(x_{0}\right)$ that are different from 1. If we choose the submanifold $Y$ such as its tangent space at $x_{0}$ is equal to $E$, we then see that the eigenvalues of the differential of $\mathcal{P}$ with respect to the first variable $x$ are the $n-1$ eigenvalues (counted with multiplicities) of $\mathrm{d} \varphi_{\tau_{u_{0}}}^{u_{0}}\left(x_{0}\right)$ that are different from 1. In conclusion, there exists $x_{u} \in X, \tau_{u}>0$ defined in a neighborhood of $u_{0}$, which depend smoothly on $u$ and which satisfy $\varphi_{\tau_{u}}^{u}\left(x_{u}, u\right)=x_{u}$.

Now the $M$-structure lemma (Lemma 31.10 in [1]) can be generalized as follows.

Lemma 14. Let $E$ be a finite dimensional Banach space. Consider $u \mapsto L_{u} a$ smooth mapping defined from a neighborhood $N$ of $u_{0} \in U$ to $\mathcal{L}(E, E)$, and $u \mapsto v_{u}$, a smooth mapping défined from $N$ to $E \backslash\{0\}$. Assume that $L_{u}$ is transversal for $v_{u}$ (i.e. $L_{u} v_{u}=v_{u}$ and the algebraic multiplicity of the eigenvalue 1 is equal to 1 ). Then for every $u \in N$, there exists $A_{u} \in \mathcal{L}(E, E)$ such that

1. $A_{u} v_{u}=0$;

2. for every mapping $\mathscr{L}: I \times U \rightarrow \mathcal{L}(E, E)$ ( $I$ denotes the interval $(0, b), b>0$ ) satisfying the three conditions

(a) $\mathscr{L}(0, u)=L_{u}$;

(b) $\left.\frac{\partial}{\partial s} \mathscr{L}(s, u)\right|_{s=0}=A_{u}$;

(c) for every $s \in I, \mathscr{L}(s, u)$ is transversal for $v_{u}$

there exists $\varepsilon>0$ and $N^{\prime} \subset N$ (with $N^{\prime}$ open neighborhood of $u_{0}$ ) such that $\mathscr{L}(s, u)$ is elementary for $v_{u}$ for every $(s, u) \in(0, \varepsilon) \times N^{\prime}$ (i.e. $\mathscr{L}(s, u)$ is transversal for $v_{u}$ and has no complex eigenvalue of modulus 1, except 1). Moreover $u \mapsto A_{u}$ is smooth.

We sketch a proof of this lemma.

Proof. We consider the set, denoted by $W$, defined as follows

$$
W=\left\{(B, u) \in L(E, E) \times N \mid B v_{u}=v_{u}\right\} .
$$

It is not difficult to see that $W$ is a submanifold of $\mathcal{L}(E, E) \times N$ of codimension $\operatorname{dim} E$ (the mapping $(B, u) \mapsto B v_{u}-v_{u}$ is a submersion).

Then, consider $M$ the subset of $\mathcal{L}(E, E)$ defined as in the proof of Lemma 31.10 in [1]. Recall that $M$ is a finite union of submanifolds of $\mathcal{L}(E, E)\left(M=\bigcup_{i=1}^{k} M_{i}\right)$, hence, $\mathcal{M} \triangleq M \times N$ is a finite union of submanifolds of $\mathcal{L}(E, E) \times N\left(\mathcal{M}=\bigcup_{i=1}^{k} M_{i} \times\right.$ $N)$; moreover every $M_{i} \times N$ has a codimension $\geq 1$ in $W$.

Denoting by $X_{u}$ the point $\left(L_{u}, u\right)$ of $W$, there exists a tangent vector $\xi_{u} \in \mathrm{T}_{X_{u}} W$ but $\xi_{u} \notin \mathrm{T}_{X_{u}} M_{i} \times N(i=1, \ldots, k)$; moreover $\xi_{u}$ depends smoothly on $u$. This is possible because each $M_{i} \times N$ has a codimension $\geq 1$ in $W$. The tangent vector $\xi_{u}$ may be written $\xi_{u}=\left(A_{u}, v\right)$, where $A_{u}$ can be regarded as an element of $\mathcal{L}(E, E)$ (and $v \in \mathrm{T}_{u} U$ ). Now, due to the property of $A_{u}$, we can conclude that there exist $\varepsilon>0$ such that $\mathscr{L}(s, u) \notin M \times N$ as soon as $0<s<\varepsilon$.

Returning to the vector field $f \in \mathscr{G}_{3 / 2}^{U}(a)$, we argue now as in Lemma 31.12, in order to prove the existence of a neighborhood $N_{1}$ of $\gamma_{u_{0}}$ and a neighborhood $N_{2}$ of $u_{0}$ such that, for every $u \in N_{2}$, the vector field $f(\cdot, u)$ admits a unique periodic trajectory $\gamma_{u}$ located in $N_{1}$. Moreover, there exists a parametrized vector field $g \in \Gamma_{U}(X)$ such that $\left.g(\cdot, u)\right|_{\gamma_{u}}=0,\left.g(\cdot, u)\right|_{X \backslash N_{1}}=0, g(\cdot, u) \equiv 0$ for $u \in U \backslash N_{2}$, and $\gamma_{u}$ is an elementary periodic orbit for $f(\cdot, u)+\lambda g(\cdot, u)$ for sufficiently small $\lambda \in \mathbf{R}_{+}^{*}$. We can find such a vector field $g$ for every periodic trajectory of $f\left(\cdot, u_{0}\right)$ (the number of these trajectories 
is finite), and so, we can conclude as in lemma 31.13 in [1], that there exists a vector field $g$ such that the critical elements of $f(\cdot, u)+\lambda g(\cdot, u)$ of period $\leq a$ are exactly the same as the critical elements of $f(\cdot, u)$ of period $\leq a$ for every $u \in N_{2}$. Moreover the periodic trajectories of $f(\cdot, u)+\lambda g(\cdot, u)$ are elementary for $u \in N_{2}$.

Now, the open neighborhoods $N_{2}$ cover $U$, which is compact. So there exist a finite set of elements $u_{0}, \ldots, u_{s}$ together with neighborhoods $N_{2}^{0}, \ldots, N_{2}^{s}$ of the $u_{i}$ 's, there exist vector fields $g_{0}, \ldots, g_{s}$ as in the above lemma. Take $\rho_{0}, \ldots, \rho_{s}$ a partition of unity of $U$ subordinated to the covering $N_{2}^{0}, \ldots, N_{2}^{s}$ and consider the vector field $g \triangleq \rho_{0} g_{0}+\cdots+\rho_{r} g_{s}$, we claim that $f+\lambda g$ belong to $\mathscr{G}_{2}^{U}(a)$ for every positive $\lambda$ sufficiently small.

We conclude this step as in [1].

Step 6: If $\mathscr{N} \subset \mathscr{G}_{1}^{U}$ is an open set, and $\mathscr{G}_{2}^{U}(a) \cap \mathscr{N}$ is dense, then $\mathscr{G}_{2}^{U}\left(\frac{3}{2} a\right) \subset \mathscr{N}$ is dense.. This step results trivially from steps 4 and 5 .

Step \%: If $f \in \mathscr{G}_{1}^{U}$, there exists an open neighborhood $\mathscr{N}$ of $f$ in $\mathscr{G}_{1}^{U}$ such that $\mathscr{G}_{2}^{U}(a) \cap \mathscr{N} \subset \mathscr{N}$ is dense.. From step 3, if $f \in \mathscr{G}_{1}^{U}$, there exists $a_{0}>0$ and a neighborhood $\mathscr{N}$ of $f$ in $\mathscr{G}_{1}^{U}$ such that $\mathscr{G}_{2}^{U}\left(a_{0}\right) \mathscr{N}=\mathscr{N}$. In particular, this implies that $\mathscr{G}_{2}^{U}\left(a_{0}\right) \mathscr{N}$ is dense in $\mathscr{N}$. Hence iterating step $5 k$ times, one has that $\mathscr{G}_{2}^{U}\left(\frac{3^{k}}{2^{k}} a_{0}\right) \cap$ $\mathscr{N}$ is dense in $\mathscr{N}$. As one can find $k$ such that $\frac{3^{k}}{2^{k}} a_{0}>a$, we are done.

\section{Appendix B. Proof of Lemma 8.}

The derivative of $x_{p}^{\lambda}$ with respect to $\lambda$ can be written as

$$
\left.\frac{\mathrm{d}}{\mathrm{d} \lambda} x_{p}^{\lambda}\right|_{\lambda=0}=A_{p}+B_{p},
$$

where $A_{p}$ is zero or a sum of terms of the form $\delta_{j_{k}}^{p}\left(J_{j_{k}}\right)$, with $J_{j_{k}}$ is an integral that can be written as

$$
J_{j_{k}}=\int_{0}^{T} \mathrm{~d} \varphi_{\sigma}^{u_{p-1}}\left(\varphi_{T-\sigma}^{u_{p-1}}\left(x_{j_{k}}\right)\right) \cdot \phi\left(\varphi_{T-\sigma}^{u_{p-1}}\left(x_{j_{k}}\right), u_{p-1}\right) \mathrm{d} \sigma,
$$

where the subscripts $j_{k}$ in $A_{p}$ are less or equal to $p-1$ and are such that $u_{j_{k}}=u_{p-1}$; moreover $\delta_{j_{k}}^{p}$ is the mapping defined as

$$
\delta_{j_{k}}^{a}= \begin{cases}\operatorname{Id} & \text { if } j_{k}=p-1 \\ \mathrm{~d}\left(\varphi_{T}^{u_{p-1}} \circ \cdots \circ \varphi_{T}^{u_{j_{k}+1}}\right)\left(x_{j_{k}+1}\right) & \text { if } j_{k}<p-1\end{cases}
$$

As for the term $B_{p}$, it is zero or the sum of terms $\delta_{j_{k}}^{p}\left(J_{j_{k}}^{\prime}\right)$, where the $J_{j_{k}}^{\prime}$ are integrals that we write

$$
J_{j_{k}}^{\prime}=\int_{0}^{T} \mathrm{~d} \varphi_{\sigma}^{u_{j_{k}}}\left(\varphi_{T-\sigma}^{u_{j_{k}}}\left(x_{j_{k}}\right)\right) \cdot \phi\left(\varphi_{T-\sigma}^{u_{j_{k}}}\left(x_{j_{k}}\right), u_{j_{k}}\right) \mathrm{d} \sigma,
$$

with $u_{j_{k}} \neq u_{p-1}$.

We write the derivative of $x_{i}^{\lambda}$ and, if we are under $\mathbf{C}_{\mathbf{2}}, \mathbf{C}_{\mathbf{3}}^{\prime}$ or $\mathbf{C}_{4}^{\prime}$ configurations, the ones of $\bar{x}_{j}^{\lambda}$ and $\bar{x}_{m}^{\lambda}$, in the same way; we denote by $\bar{J}_{j_{k}}$ and $\bar{J}_{j_{k}}^{\prime}$ the integrals appearing in the derivatives of $\bar{x}_{j}^{\lambda}$ and $\bar{x}_{m}^{\lambda}$. We shall see that $\phi$ can be chosen such that

- the terms $B_{p}, B_{i}, \bar{B}_{j}$ and $\bar{B}_{m}$ are zero;

- all the integrals $\bar{J}_{j_{k}}$ that occur in the terms $\bar{A}_{j}$ and $\bar{A}_{m}$ are zero;

- all the integrals $J_{j_{k}}$ are zero but the one corresponding to the subscript $j_{k}=$ $p-1$, which can be arbitrarily chosen. 
To this end, the perturbation $\phi$ that we shall consider will be zero outside some neighborhood of $u_{p-1}$; to be more precise, given $\phi_{0}$ a vector field defined on $X$, it is possible to find $\phi \in \Gamma_{U}(X)$ such that

- $\phi\left(\cdot, u_{p-1}\right)=\phi_{0}$;

- for $j=0, \ldots, 2 n, \phi\left(\cdot, u_{j}\right) \equiv 0$ as soon as $u_{j} \neq u_{p-1}$.

With this choice of $\phi$, we have $B_{p}=0$, and $B_{i}=0$ as well as $\bar{B}_{j}=0$, and $\bar{B}_{m}=0$ if we are under $\mathbf{C}_{\mathbf{2}}, \mathbf{C}_{\mathbf{3}}^{\prime}$ or $\mathbf{C}_{\mathbf{4}}^{\prime}$ configuration.

We split the points $x_{j_{k}}$ (resp. $\bar{x}_{j_{k}}$ ) that appear under the integrals $J_{j_{k}}$ (resp. $\left.\bar{J}_{j_{k}}\right)$ into two classes: the first class, denoted by $\mathscr{P}_{1}$, contains the points $x_{j_{k}}$ and $\bar{x}_{j_{k}}$ that belong to the trajectory of the vector field $f\left(\cdot, u_{p-1}\right)$ passing through $x_{p-1}$ (and so $\mathscr{P}_{1}$ contains the point $x_{p-1}$ itself), the second class, denoted by $\mathscr{P}_{2}$, contains the points $x_{j_{k}}$ and $\bar{x}_{j_{k}}$ that do not belong to this trajectory. Denote by $\mathscr{T}_{1}$ and $\mathscr{T}_{2}$ the union of these trajectories restricted to the interval $[0, T]$, namely

$$
\mathscr{T}_{i}=\left\{\varphi_{t}^{u_{p-1}}(z) \mid t \in[0, T], z \in \mathscr{P}_{i}\right\} \quad i=1,2 .
$$

The sets $\mathscr{T}_{1}$ and $\mathscr{T}_{2}$ being disjoint and compact, let $\mathscr{U}_{1}$ and $\mathscr{U}_{2}$ be two open sets of $X$ such that $\mathscr{T}_{i} \subset \mathscr{U}_{i}(i=1,2)$ and $\mathscr{U}_{1} \cap \mathscr{U}_{2}=\varnothing$. In the sequel, we shall assume that the vector field $\phi_{0}$ is zero when restricted to $\mathscr{U}_{2}$; this implies that the integrals $J_{j_{k}}$ (resp. $\bar{J}_{j_{k}}$ ) such that $x_{j_{k}}$ (resp. $\bar{x}_{j_{k}}$ ) belongs to $\mathscr{P}_{2}$ are zero. Denote by $j_{1}, \ldots, j_{a}\left(0 \leq j_{1}<\cdots<j_{a}=p-1\right)$ the subscripts such that $x_{j_{k}}$ belongs to $\mathscr{P}_{1}$ and let $t_{1}, \ldots, t_{a}$ be such that $x_{j_{k}}=\varphi_{t_{k}}^{u_{p-1}}\left(x_{p-1}\right)(k=1, \ldots, a)$. Denote also by $l_{1}<\cdots<l_{b}<\max (m, j)$ the subscripts such that $\bar{x}_{l_{k}}$ belongs to $\mathscr{P}_{1}$ and let $t_{1}^{\prime}, \ldots, t_{b}^{\prime}$ be such that $\bar{x}_{l_{k}}=\varphi_{t_{k}^{\prime}}^{u_{p-1}}\left(x_{p-1}\right)(k=1, \ldots, b)$.

Notice that, excepted when $j_{k}=j_{a}$, we cannot have $t_{k}=0$ since this would imply that $x_{p-1}=x_{j_{k}}$ with $j_{k}<p-1$, which is not possible under $\mathbf{C}_{\mathbf{1}}-\mathbf{C}_{\mathbf{4}}$ configuration. Also, all the $t_{k}^{\prime}(k=1, \ldots, b)$ are non zero, because, if there existed a subscript $k$ such that $t_{k}^{\prime}=0$, then, as $u_{l_{k}}=u_{p-1}$, we would have $\bar{x}_{l_{k}+1}=x_{p}$, which implies that we are under $\mathbf{C}_{\mathbf{2}}$ or $\mathbf{C}_{\mathbf{3}}^{\prime}$ configuration and that $l_{k}+1=m$. Thus we have $\bar{x}_{m-1}=x_{p-1}$, if we are under $\mathbf{C}_{\mathbf{2}}^{\prime}$ configuration this implies $(m-1)-(p-1)=i-j$ and so $m-p=i-j$, which contradicts the definition of the $\mathbf{C}_{\mathbf{2}}$ configuration. If we are under $\mathbf{C}_{\mathbf{3}}^{\prime}$ configuration, we found a pair $\left(i^{\prime}, j^{\prime}\right) \triangleq(p-1, m-1)$ such that $x_{i^{\prime}}=\bar{x}_{j^{\prime}}$ with $i^{\prime}-j^{\prime}=p-m$ and $i^{\prime}<p$, which contradicts the definition of $\mathbf{C}_{\mathbf{3}}$ configuration.

The trajectory related to the vector field $f\left(\cdot, u_{p-1}\right)$ passing through $x_{p-1}$ may be periodic or aperiodic, we have to distinguish between these two cases.

The trajectory passing through $\boldsymbol{x}_{\boldsymbol{p}-1}$ is not periodic. Assume that the trajectory of the vector field $f\left(\cdot, u_{p-1}\right)$ passing through $x_{p-1}$ is not periodic. Taking into account that, with our notations, $\phi\left(\cdot, u_{p-1}\right)=\phi_{0}$, the terms $J_{j_{k}}$ appearing in $A_{p}$ and (possibly) in $A_{i}$ can also be written as

$$
J_{j_{k}}=\mathrm{d} \varphi_{t_{k}}^{u_{p-1}}\left(x_{p}\right) \cdot \int_{-t_{k}}^{T-t_{k}} \mathrm{~d} \varphi_{\sigma}^{u_{p-1}} \circ \phi_{0} \circ \varphi_{T-\sigma}^{u_{p-1}}\left(x_{p-1}\right) \mathrm{d} \sigma .
$$

In the same way the integrals $\bar{J}_{l_{k}}$ appearing (possibly) in $\bar{A}_{m}$ and $\bar{A}_{j}$ write

$$
\bar{J}_{l_{k}}=\mathrm{d} \varphi_{t_{k}^{\prime}}^{u_{p-1}}\left(x_{p}\right) \cdot \int_{-t_{k}^{\prime}}^{T-t_{k}^{\prime}} \mathrm{d} \varphi_{\sigma}^{u_{p-1}} \circ \phi_{0} \circ \varphi_{T-\sigma}^{u_{p-1}}\left(x_{p-1}\right) \mathrm{d} \sigma .
$$


We set

$$
\begin{aligned}
T_{\min } & =\min \left(\left\{t_{k} \mid k=1, \ldots, a\right\} \cup\left\{t_{k}^{\prime} \mid k=1, \ldots, b\right\}\right) \\
T_{\max } & =\max \left(\left\{T+t_{k} \mid k=1, \ldots, a\right\} \cup\left\{T+t_{k}^{\prime} \mid k=1, \ldots, b\right\}\right)
\end{aligned}
$$

and we introduce the set $\mathcal{T}=\left\{\varphi_{t}^{u_{p-1}}\left(x_{p-1}\right) \mid T_{\min } \leq t \leq T_{\max }\right\}$. For $z=\varphi_{t}^{u_{p-1}}\left(x_{p-1}\right)$ in $\mathcal{T}$, we define $\phi_{0}(z)$ as

$$
\phi_{0}(z)=\mu(t) \mathrm{d} \varphi_{t-T}^{u_{p-1}}\left(x_{p}\right) \cdot \mathfrak{X}_{p}
$$

where $\mu$ is a smooth function defined on $\left[T_{\min }, T_{\max }\right]$ and $\mathfrak{X}_{p}$ is an arbitrary vector tangent to $X$ at $x_{p}$. As the trajectory passing through $x_{p-1}$ is not periodic, $\phi_{0}$ is unambiguously defined on $\mathcal{T}$, moreover $\phi_{0}$ extends to a smooth vector field defined on the whole manifold $X$ (and which is zero on $\mathscr{U}_{2}$ ). With this choice of $\phi_{0}$, the integrals occurring in $A_{i}$ and $A_{p}$ write

$$
J_{j_{k}}=\left(\int_{-t_{k}}^{T-t_{k}} \mu(T-\sigma) \mathrm{d} \sigma\right) \mathrm{d} \varphi_{t_{k}}^{u_{p-1}}\left(x_{p}\right) \cdot \mathfrak{X}_{p}=\left(\int_{t_{k}}^{T+t_{k}} \mu(\sigma) \mathrm{d} \sigma\right) \mathrm{d} \varphi_{t_{k}}^{u_{p-1}}\left(x_{p}\right) \cdot \mathfrak{X}_{p} ;
$$

while the integrals occurring in the terms $\bar{A}_{j}$ and $\bar{A}_{m}$ write

$$
\bar{J}_{l_{k}}=\left(\int_{-t_{k}^{\prime}}^{T-t_{k}^{\prime}} \mu(T-\sigma) \mathrm{d} \sigma\right) \mathrm{d} \varphi_{t_{k}^{\prime}}^{u_{p-1}}\left(x_{p}\right) \cdot \mathfrak{X}_{p}=\left(\int_{t_{k}^{\prime}}^{T+t_{k}^{\prime}} \mu(\sigma) \mathrm{d} \sigma\right) \mathrm{d} \varphi_{t_{k}^{\prime}}^{u_{p-1}}\left(x_{p}\right) \cdot \mathfrak{X}_{p} ;
$$

here all the $t_{k}$ 's are non-zero but $t_{a}$, and all the $t_{k}^{\prime}$ 's are non zero. Choose now a smooth function $M$ defined on $\left[T_{\min }, T_{\max }\right]$ and such that

- $M(T)=M(2 T)=\cdots=M(c T)=1$; here $c$ denotes the integer part of $T_{\max } / T$

- $M\left(T+t_{k}\right)=M\left(t_{k}\right)=M\left(T+t_{k^{\prime}}^{\prime}\right)=M\left(t_{k^{\prime}}^{\prime}\right)=0, k=1, \ldots, a-1, k^{\prime}=1, \ldots, b$ where $t_{k}, t_{k}^{\prime} \neq \alpha T, \alpha=1, \ldots, c$;

- and $M(0)=0$;

We take now $\mu(t)=\frac{\mathrm{d} M(t)}{\mathrm{d} t}$, with this choice of $\mu$, all the integrals $J_{j_{k}}$ and $\bar{J}_{l_{k}}$ are zero except $J_{p-1}$ which is equal to $\mathfrak{X}_{p}$.

Point $x_{p-1}$ is singular.. In this case, we have $x_{p-1}=x_{p}$, so necessarily we are under $\mathbf{C}_{\mathbf{1}}$ or $\mathbf{C}_{\mathbf{4}}^{\prime}$ configuration. The vector field $\phi_{0}$ is then chosen such that $\phi_{0}$ is zero outside an open neighborhood $\mathcal{N}$ of $x_{p-1}$, this neighborhood being chosen so small that the trajectories of $f\left(\cdot, u_{p-1}\right)$ passing through the points $x_{j_{k}}\left(j_{k} \neq p-1\right)$ and $\bar{x}_{l_{k}}$ do not cross $\mathcal{N}$. With this choice of $\phi_{0}$ all the integrals $J_{j_{k}}$ and $\bar{J}_{l_{k}}$ are zero but the one which correspond to $j_{k}=p-1$ which is equal to

$$
J_{p-1}=\int_{0}^{T} \mathrm{~d} \varphi_{s}^{u_{p-1}}\left(x_{p-1}\right) \cdot \phi_{0}\left(x_{p-1}\right) \mathrm{d} s .
$$

Now $\mathrm{d} \varphi_{s}^{u_{p-1}}\left(x_{p-1}\right)=e^{s A}$ where $A$ is the differential of $f$ at $x_{p-1}$; notice that, as $f \in \mathscr{G}_{2}^{U}(a), A$ does not have any purely imaginary eigenvalue (and so is invertible). Hence we can compute explicitly integral $J_{p-1}$ :

$$
J_{p-1}=A^{-1}\left(e^{T A}-\mathrm{Id}\right) \cdot \phi_{0}\left(x_{p-1}\right) ;
$$

as $e^{T A}$ does not admit 1 as an eigenvalue, $e^{T A}-$ Id is invertible, which proves that $J_{p-1}$ can be made equal to any tangent vector of $T_{x_{p-1}}$ thanks to an appropriate choice of $\phi_{0}\left(x_{p-1}\right)$. 
The trajectory passing through $\boldsymbol{x}_{\boldsymbol{p}-\mathbf{1}}$ is periodic. We shall show now the same result in the case when the trajectory passing through $x_{p-1}$ is periodic; in other words, we assume that the mapping $t \mapsto \varphi_{t}^{u_{p-1}}\left(x_{p-1}\right)$ is periodic and we denote by $\pi_{0}$ its prime period. In this case, the function $\mu$ which appears in the definition of $\phi_{0}$ must be periodic. Writing $T=q \pi_{0}+\tau$ with $q \in \mathbf{N}$ and $0 \leq \tau<\pi_{0}$, we have

$$
\begin{aligned}
J_{j_{k}}= & \int_{0}^{T} \mathrm{~d} \varphi_{\sigma}^{u_{p-1}} \circ \phi_{0} \circ \varphi_{T-\sigma}^{u_{p-1}}\left(x_{j_{k}}\right) \mathrm{d} \sigma \\
= & \sum_{l=0}^{q-1} \int_{l \pi_{0}}^{(l+1) \pi_{0}} \mathrm{~d} \varphi_{\sigma}^{u_{p-1}} \circ \phi_{0} \circ \varphi_{T-\sigma}^{u_{p-1}}\left(x_{j_{k}}\right) \mathrm{d} \sigma+\int_{q \pi_{0}}^{T} \mathrm{~d} \varphi_{\sigma}^{u_{p-1}} \circ \phi_{0} \circ \varphi_{T-\sigma}^{u_{p-1}}\left(x_{j_{k}}\right) \mathrm{d} \sigma \\
= & \sum_{l=0}^{q-1} \mathrm{~d} \varphi_{l \pi_{0}}^{u_{p-1}}\left(\varphi_{T}^{u_{p-1}}\left(x_{j_{k}}\right)\right) \cdot \int_{0}^{\pi_{0}} \mathrm{~d} \varphi_{\sigma}^{u_{p-1}} \circ \phi_{0} \circ \varphi_{T-\sigma}^{u_{p-1}}\left(x_{j_{k}}\right) \mathrm{d} \sigma \\
& \quad+\mathrm{d} \varphi_{q \pi_{0}}^{u_{p-1}}\left(\varphi_{T}^{u_{p-1}}\left(x_{j_{k}}\right)\right) \int_{0}^{\tau} \mathrm{d} \varphi_{\sigma}^{u_{p-1}} \circ \phi_{0} \circ \varphi_{T-\sigma}^{u_{p-1}}\left(x_{j_{k}}\right) \mathrm{d} \sigma .
\end{aligned}
$$

Now the $x_{j_{k}}$ 's in the above integrals are such that $x_{j_{k}}=\varphi_{t_{k}}^{u_{p-1}}\left(x_{p-1}\right)$. Notice that, due to the periodicity of the trajectory passing through $x_{p-1}$, we can assume that $0 \leq t_{k}<\pi_{0}$. Writing the $x_{j_{k}}$ 's in terms of $x_{p-1}$, the above integrals between 0 and $\pi_{0}$ can be re-written as.

$$
\int_{0}^{\pi_{0}} \mathrm{~d} \varphi_{\sigma}^{u_{p-1}} \circ \phi_{0} \circ \varphi_{T-\sigma}^{u_{p-1}}\left(x_{j_{k}}\right) \mathrm{d} \sigma=\mathrm{d} \varphi_{t_{k}}^{u_{p-1}}\left(x_{p}\right) \cdot \int_{-t_{k}}^{\pi_{0}-t_{k}} \mathrm{~d} \varphi_{\sigma}^{u_{p-1}} \circ \phi_{0} \circ \varphi_{T-\sigma}^{u_{p-1}}\left(x_{p-1}\right) \mathrm{d} \sigma .
$$

In the same way, the integral between 0 and $\tau$ in (28) can be written

$$
\int_{0}^{\tau} \mathrm{d} \varphi_{\sigma}^{u_{p-1}} \circ \phi_{0} \circ \varphi_{T-\sigma}^{u_{p-1}}\left(x_{j_{k}}\right) \mathrm{d} \sigma=\mathrm{d} \varphi_{t_{k}}^{u_{p-1}}\left(x_{p}\right) \int_{-t_{k}}^{\tau-t_{k}} \mathrm{~d} \varphi_{\sigma}^{u_{p-1}} \circ \phi_{0} \circ \varphi_{T-\sigma}^{u_{p-1}}\left(x_{p-1}\right) \mathrm{d} \sigma .
$$

It follows from these considerations and from the equality $\varphi_{T}^{u_{p-1}}\left(x_{p-1}\right)=x_{p}$, that we can write $J_{j_{k}}$ under the form

$$
\begin{array}{r}
J_{j_{k}}=\sum_{l=0}^{q-1} \mathrm{~d} \varphi_{l \pi_{0}+t_{k}}^{u_{p-1}}\left(x_{p}\right) \cdot \int_{-t_{k}}^{\pi_{0}-t_{k}} \mathrm{~d} \varphi_{\sigma}^{u_{p-1}} \circ \phi_{0} \circ \varphi_{-\sigma}^{u_{p-1}}\left(x_{p}\right) \mathrm{d} \sigma \\
+\mathrm{d} \varphi_{q \pi_{0}+t_{k}}^{u_{p-1}}\left(x_{p}\right) \cdot \int_{-t_{k}}^{\tau-t_{k}} \mathrm{~d} \varphi_{\sigma}^{u_{p-1}} \circ \phi_{0} \circ \varphi_{-\sigma}^{u_{p-1}}\left(x_{p}\right) \mathrm{d} \sigma \\
=\mathrm{d} \varphi_{t_{k}}^{u_{p-1}}\left(x_{p}\right) \cdot\left(Q \cdot \int_{-t_{k}}^{\pi_{0}-t_{k}} \mathrm{~d} \varphi_{\sigma}^{u_{p-1}} \circ \phi_{0} \circ \varphi_{-\sigma}^{u_{p-1}}\left(x_{p}\right) \mathrm{d} \sigma\right. \\
\left.\quad+\delta^{q} \cdot \int_{-t_{k}}^{\tau-t_{k}} \mathrm{~d} \varphi_{\sigma}^{u_{p-1}} \circ \phi_{0} \circ \varphi_{-\sigma}^{u_{p-1}}\left(x_{p}\right) \mathrm{d} \sigma\right) ;
\end{array}
$$

where we let

$$
\delta=\mathrm{d} \varphi_{\pi_{0}}^{u_{p-1}}\left(x_{p}\right) \quad \text { and } \quad Q=\sum_{l=0}^{q-1} \delta^{l} .
$$

We introduce also the following notation, let $t \in\left[0, \pi_{0}\right]$ and denote by $I_{t}$ the integral

$$
I_{t}=\int_{0}^{t} \mathrm{~d} \varphi_{\sigma}^{u_{p-1}} \circ \phi_{0} \circ \varphi_{-\sigma}^{u_{p-1}}\left(x_{p}\right) \mathrm{d} \sigma .
$$


We now rewrite $J_{j_{k}}$ in terms of integrals $I_{t}$, from (29), we get

- if $t_{k}<\tau$,

(31) $J_{j_{k}}=\mathrm{d} \varphi_{t_{k}}^{u_{p-1}}\left(x_{p}\right) \cdot\left(\left(Q \circ \delta^{-1}+\delta^{q-1}\right) \cdot I_{\pi_{0}}-\delta^{-1} \cdot I_{\pi_{0}-t_{k}}+\delta^{q} \cdot I_{\tau-t_{k}}\right)$.

- if $\tau \leq t_{k}$

$$
J_{j_{k}}=\mathrm{d} \varphi_{t_{k}}^{u_{p-1}}\left(x_{p}\right) \cdot\left(Q \circ \delta^{-1} \cdot I_{\pi_{0}}-\delta^{-1} \cdot I_{\pi_{0}-t_{k}}+\delta^{q-1} \cdot I_{\pi_{0}+\tau-t_{k}}\right)
$$

notice that, in each case, $\pi_{0}-t_{k}$ and $\pi_{0}+\tau-t_{k}$ belong to $\left[0, \pi_{0}\right]$;

As regards the integrals $\bar{J}_{l_{k}}(k=1, \ldots, b)$, analogous computations lead to the same above formulas (31) and (32).

We choose now $\phi_{0}$ as follows, for $z=\varphi_{-\sigma}^{u_{p-1}}\left(x_{p}\right)$ with $\sigma \in\left[0, \pi_{0}\right]$, we define $\phi_{0}(z)$ as

$$
\phi_{0}(z)=\mathrm{d} \varphi_{-\sigma}^{u_{p-1}}\left(x_{p}\right) \cdot \vartheta(\sigma)
$$

where $\vartheta: \mathbf{R} \rightarrow \mathrm{T}_{x_{p}} X$ is a $\pi_{0}$-periodic mapping to be determined; notice first that, as we want $\phi_{0}$ to be $C^{r}$, we must have $\vartheta(0)=\vartheta\left(\pi_{0}\right)=0$ as well as $\vartheta^{(l)}(0)=\vartheta^{(l)}\left(\pi_{0}\right)=0$ for $l=1, \ldots, r$.

We deal first with the special case $\tau=0$, in this case $T=q \pi_{0}$ and we have $x_{p-1}=x_{p}$, so the lists $L_{p}$ and $\bar{L}_{m}$ must be in the $\mathbf{C}_{\mathbf{1}}$ or $\mathbf{C}_{\mathbf{4}}^{\prime}$ configuration. If we are under $\mathbf{C}_{4}^{\prime}$ configuration and if there exists a subscript $j_{1}<p-1$ such that $u_{j_{1}}=u_{p-1}$ and $x_{j_{1}}=\varphi_{t_{1}}^{u_{p-1}}\left(x_{p-1}\right)$, then $x_{j_{1}}=x_{j_{1}+1}$ which is impossible from the definition of the $\mathbf{C}_{\mathbf{4}}^{\prime}$ configuration. If there exists a subscript $l_{1}<m$ such that $u_{l_{1}}=u_{p-1}$ and $\bar{x}_{l_{1}}=\varphi_{t_{1}^{\prime}}^{u_{p-1}}\left(x_{p-1}\right)$, then we would have $\bar{x}_{l_{1}}=\bar{x}_{l_{1}+1}$; as the lists $L_{p}$ and $\bar{L}_{m}$ are in the $\mathbf{C}_{4}$ configuration, this implies that $l_{1}=m-1$ and $u_{m-1}=u_{p-1}$ which is incompatible with the definition of the $\mathbf{C}_{\mathbf{4}}^{\prime}$ configuration. We conclude that the terms $A_{p-1}, \bar{A}_{j}$ and $\bar{A}_{m}$ are zero in this case and that $A_{p}$ is equal to $Q \cdot I_{\pi_{0}}$; so, with our choice of $\phi_{0}$, we have

$$
A_{p}=Q \cdot \int_{0}^{\pi_{0}} \vartheta(\sigma) \mathrm{d} \sigma
$$

and it is obviously possible to find a periodic function $\vartheta$ satisfying the above constraints and whose integral over the interval $\left[0, \pi_{0}\right]$ is equal to $Q^{-1} \cdot \mathfrak{X}_{p}$; clearly this choice of $\vartheta$ is also possible if we are under $\mathbf{C}_{\mathbf{1}}$ configuration.

We assume now that $\tau \neq 0$; we deal first with the case of $\mathbf{C}_{\mathbf{1}}$ configuration. We introduce the following sets.

$$
\mathbf{T}_{\alpha}=\left\{t_{k}, k=1, \ldots, a-1 \mid t_{k} \equiv-\alpha \tau \quad\left(\bmod \pi_{0}\right)\right\} .
$$

If $\mathbf{T}_{1}=\varnothing$, we set $\alpha_{0}=0$, if not, we denote by $\alpha_{0}$ the largest integer $\alpha$ such that $\mathbf{T}_{1} \neq \varnothing, \mathbf{T}_{2} \neq \varnothing, \ldots, \mathbf{T}_{\alpha} \neq \varnothing$. If $\alpha_{0} \neq 0$, we introduce the integers $\gamma_{\alpha}=\left[\alpha \tau / \pi_{0}\right]+1$ (where $[x]$ denotes the integer part of $x$ ); so if $t_{k} \in \mathbf{T}_{\alpha}$, we have $t_{k}=\gamma_{\alpha} \pi_{0}-\alpha \tau$.

It could happen that $\pi_{0}$ is divisible by $\tau$; hereafter, we distinguish two cases.

First we assume that there does not exist any $\alpha \leq \alpha_{0}$ such that $(\alpha+1) \tau \equiv 0$ $\left(\bmod \pi_{0}\right)$; this assumption implies that, for every pair $0 \leq \alpha<\alpha^{\prime} \leq \alpha_{0}$ we have $\alpha^{\prime} \tau-\alpha \tau \not \equiv 0\left(\bmod \pi_{0}\right)$. Thus, there exists a mapping $V: \mathbf{R} \rightarrow \mathrm{T}_{x_{p}} X$ such that

- $V$ is $\pi_{0}$-periodic and $V(0)=0, V^{(l)}(0)=0$ for $l=1, \ldots, r+1$; 
- $V(\tau)=\delta^{-q} \cdot \mathfrak{X}_{p}$, and

$$
V(2 \tau)= \begin{cases}\delta^{-q-1} \cdot V(\tau) & \text { if } \pi_{0}<2 \tau \\ \delta^{-q} \cdot V(\tau) & \text { if } \pi_{0}>2 \tau\end{cases}
$$

$\vdots$

$$
V\left(\left(\alpha_{0}+1\right) \tau\right)= \begin{cases}\delta^{-q-1} \cdot V\left(\alpha_{0} \tau\right) & \text { if } \gamma_{\alpha_{0}} \pi_{0}<\left(\alpha_{0}+1\right) \tau \\ \delta^{-q} \cdot V\left(\alpha_{0} \tau\right) & \text { if } \gamma_{\alpha_{0}} \pi_{0}>\left(\alpha_{0}+1\right) \tau\end{cases}
$$

- $V\left(\pi_{0}-t_{k}\right)=0, V\left(\tau-t_{k}\right)=0\left(\right.$ case $\left.t_{k}<\tau\right)$ or $V\left(\pi_{0}+\tau-t_{k}\right)=0$ (case $\tau \leq t_{k}$ ) if $t_{k} \notin \mathbb{T}_{1} \cup \cdots \cup \mathbb{T}_{\alpha_{0}}$ (notice that, for such a $t_{k}, \pi_{0}-t_{k}$ and $\pi_{0}+\tau-t_{k}$ do no more belong to $\mathbb{T}_{1} \cup \cdots \cup \mathbb{T}_{\alpha_{0}}$ ).

We take then the mapping $\vartheta$ equal to the derivative of $V$, with this choice, all the integrals $J_{j_{k}}$ are zero but $J_{p-1}$ which is equal to $\mathfrak{X}_{p}$; this implies that $A_{p}$ is equal to $\mathfrak{X}_{p}$ while we have $A_{i}=0$.

Case where $\tau$ divides $\pi_{0}$. Assume now that there exists $\alpha \leq \alpha_{0}$ such that $(\alpha+$ 1) $\tau \equiv 0\left(\bmod \pi_{0}\right)$, then necessarily $\alpha=\alpha_{0}$ (if $\alpha<\alpha_{0}$, the time $t_{a}=0$ would belong to $\left.\mathbf{T}_{\alpha+1}\right)$. There exists a subscript $j_{k}$ such that $x_{j_{k}}=\varphi_{-\alpha_{0} \tau}^{u_{p-1}}\left(x_{p-1}\right)=\varphi_{\tau}^{u_{p-1}}\left(x_{p-1}\right)=$ $x_{p}$, as $j_{k}<p$, from this equality and from the definition of configuration $\mathbf{C}_{\mathbf{1}}$, we deduce that $j_{k}=i$ and that $u_{i}=u_{p-1}$. Arguing by induction, assume that, for some $0 \leq r<p-1-i$, we have $u_{i}=\cdots=u_{i+r}=u_{p-1}$ and $x_{i}=\varphi_{-\alpha_{0} \tau}^{u_{p-1}}\left(x_{p-1}\right), \ldots, x_{i+r}=$ $\varphi_{-\left(\alpha_{0}-r\right) \tau}^{u_{p-1}}\left(x_{p-1}\right)$. There exists a subscript $j_{k}$ such that $x_{j_{k}}=\varphi_{-\left(\alpha_{0}-r-1\right) \tau}^{u_{p-1}}\left(x_{p-1}\right)=$ $\varphi_{\tau}^{u_{p-1}}\left(x_{i+r}\right)=x_{i+r+1}$, as above, this equality implies that $u_{i+r+1}=u_{p-1}$. We have proved that $u_{i}=u_{i+1}=\cdots=u_{p-1}$ and that $\alpha_{0}=p-i-1$. As $x_{p}=\varphi_{(p-i) T}^{u_{p-1}}\left(x_{i}\right)$, we re-write $A_{p}$ as follows:

$$
A_{p}=\int_{0}^{(p-i) T} \mathrm{~d} \varphi_{\sigma}^{u_{p-1}} \circ \phi_{0} \circ \varphi_{-\sigma}^{u_{p-1}}\left(x_{p}\right) \mathrm{d} \sigma+\mathrm{d} \varphi_{(p-i) T}^{u_{p-1}}\left(x_{p}\right) \cdot A_{i} .
$$

As $\left(\alpha_{0}+1\right) \tau \equiv 0\left(\bmod \pi_{0}\right)$, there exists $r \in \mathbf{N}$ such that $(p-i) T=q^{\prime} \pi_{0}$, thus the first term in this new expression of $A_{p}$ can be re-written as

$$
\int_{0}^{(p-i) T} \mathrm{~d} \varphi_{\sigma}^{u_{p-1}} \circ \phi_{0} \circ \varphi_{-\sigma}^{u_{p-1}}\left(x_{p}\right) \mathrm{d} \sigma=Q^{\prime} \cdot I_{\pi_{0}}
$$

where $Q^{\prime}=\operatorname{Id}+\delta+\ldots \delta^{q^{\prime}-1}$. We shall see that $\phi_{0}$ can be chosen such that $A_{i}=0$ and $A_{p}$ equal to any tangent vector field. Hereafter, we call chain of length $c(c \geq 2)$ a sequence of $c$ pairs $\left(\left(x_{j_{k_{1}}}, t_{k_{1}}\right), \ldots,\left(x_{j_{k_{c}}}, t_{k_{c}}\right)\right)$ such that

- the points $x_{j_{k_{1}}}, \ldots, x_{j_{k_{c}}}$ belong to $\mathscr{P}_{1}$ and the subscripts $j_{k_{1}}, \ldots j_{k_{c}}$ are pairwise distinct;

- the times $t_{k_{1}}, \ldots, t_{k_{c}}$ belong to $\mathbf{T}_{1} \cup \cdots \cup \mathbf{T}_{\alpha_{0}} \cup\{0\}$ and are such that

$$
t_{k_{2}} \equiv t_{k_{1}}+\tau \quad\left(\bmod \pi_{0}\right), \ldots, t_{k_{c}} \equiv t_{k_{1}}+(c-1) \tau \quad\left(\bmod \pi_{0}\right) .
$$

Notice that two chains are either disjoint or equal. The chain

$$
\mathfrak{c}_{0} \triangleq\left(\left(x_{i}, \tau\right),\left(x_{i+1}, 2 \tau\right), \ldots,\left(x_{p-1},\left(\alpha_{0}+1\right) \tau\right)\right)
$$

has a length equal to $\alpha_{0}+1$; the lengths of all the other chains are less than $\alpha_{0}+1$ because otherwise, we could find at least two equalities between the elements of $L_{p}$. 
We choose vector field $\phi_{0}$ as in the case where $\left(\alpha_{0}+1\right) \tau \not \equiv 0\left(\bmod \pi_{0}\right)$, but the mappings $\vartheta$ and $V$ are chosen as follows:

- $\vartheta(t)=V^{\prime}(t)$ and $V(t)=V_{0}(t)+t v_{0}$ where $V_{0}$ is $\pi_{0}$-periodic with $V_{0}\left(\pi_{0}\right)=$ $V_{0}(0)=0$; notice that this choice of $\vartheta$ implies $I_{t}=V_{0}(t)+t v_{0}$;

- $V_{0}^{\prime}(0)=V_{0}^{\prime}\left(\pi_{0}\right)=-v_{0}$ and $V_{0}^{(k)}=V_{0}^{(k)}\left(\pi_{0}\right)=0$ with $k=2, \ldots, r+1$;

- $v_{0}=\frac{1}{\pi_{0}} \cdot\left(Q^{\prime}\right)^{-1} \mathfrak{X}_{p}$;

- if $\left(\left(x_{j_{k_{1}}}, t_{k_{1}}\right), \ldots,\left(x_{j_{k_{c}}}, t_{k_{c}}\right)\right)$ is a chain of maximal length distinct from the chain $\mathfrak{c}_{0}$, we must have

$$
\left\{\begin{array}{ll}
\left(Q \circ \delta^{-1}+\delta^{q-1}\right) \cdot I_{\pi_{0}}-\delta^{-1} \cdot I_{\pi_{0}-t_{k_{s}}}+\delta^{q} \cdot I_{\tau-t_{k_{s}}}=0, & \text { if } t_{k_{s}}<\tau \\
Q \circ \delta^{-1} \cdot I_{\pi_{0}}-\delta^{-1} \cdot I_{\pi_{0}-t_{k_{s}}}+\delta^{q-1} \cdot I_{\pi_{0}+\tau-t_{k_{s}}}=0, & \text { if } \tau \leq t_{k_{s}}
\end{array} .\right.
$$

These equalities can also be written as

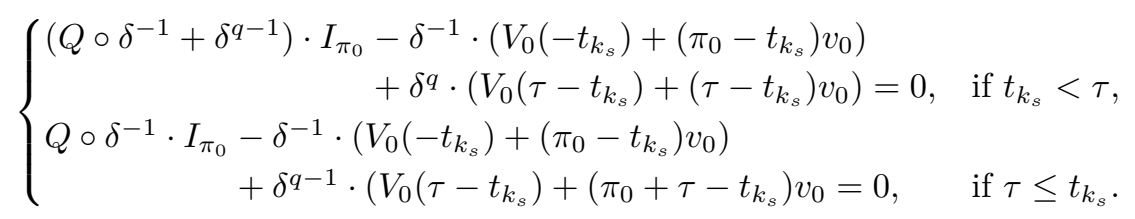

As $c<\alpha_{0}+1$, the numbers $\tau-t_{k_{1}},-t_{k_{1}}, \ldots,-t_{k_{1}}-(c-1) \tau$ are pairwise distinct modulo $\pi_{0}$, the values of $V_{0}$ at these points can be chosen independently from each other; this proves that the above equalities can be achieved.

- If $t_{k}$ does not belong to a chain, nor is the case for $\tau-t_{k}$, so equality (34) can be satisfied thanks to an appropriate choice of $V\left(-t_{k}\right)$ and $V\left(\tau-t_{k}\right)$.

With this choice of $\vartheta$, taking into account the formulas (31) and (32), we see that we have $A_{p}=\mathfrak{X}_{p}$ and $A_{i}=0$.

If we are under the configurations $\mathbf{C}_{\mathbf{2}}, \mathbf{C}_{\mathbf{3}}^{\prime}$ or $\mathbf{C}_{\mathbf{4}}^{\prime}$, the sets $\mathbf{T}_{\alpha}$ are defined as follows.

$$
\begin{aligned}
& \mathbf{T}_{\alpha}=\left\{t_{k}, k=1, \ldots, a-1 \mid t_{k} \equiv-\alpha \tau\right.\left.\left(\bmod \pi_{0}\right)\right\} \\
& \cup\left\{t_{k}^{\prime}, k=1, \ldots, b \mid t_{k}^{\prime} \equiv-\alpha \tau\left(\bmod \pi_{0}\right)\right\},
\end{aligned}
$$

the integer $\alpha_{0}$ being defined as above. If there does not exist any $\alpha \leq \alpha_{0}$ such that $(\alpha+1) \tau \equiv 0\left(\bmod \pi_{0}\right)$, the reasoning is exactly the same than in the the case of $\mathbf{C}_{\mathbf{1}}$ configuration: choosing $\phi_{0}$ as explained above, we get $A_{i}=0, \bar{A}_{m}=0$ and $\bar{A}_{j}=0$ while $A_{p}$ can be arbitrarily chosen.

Assume now that there exists $\alpha$ such that $(\alpha+1) \tau \equiv 0\left(\bmod \pi_{0}\right)$, then, as above, necessarily $\alpha=\alpha_{0}$. As we are under $\mathbf{C}_{2}, \mathbf{C}_{3}^{\prime}$ or $\mathbf{C}_{4}^{\prime}$ configuration, the definition of a chain takes into account the elements of $\bar{L}_{2 n}$ : we call chain of length $c$ a sequence $\left(\left(z_{1}, \mathfrak{t}_{k_{1}}\right), \ldots,\left(z_{c}, \mathfrak{t}_{k_{c}}\right)\right)$ such that

- the points $z_{1}, \ldots, z_{c}$ belong to $\mathscr{P}_{1}$;

- the times $\mathfrak{t}_{k_{1}}, \ldots, \mathfrak{t}_{k_{c}}$ belong to $\mathbf{T}_{1} \cup \cdots \cup \mathbf{T}_{\alpha_{0}} \cup\{0\}$ and are such that

$$
\mathfrak{t}_{k_{2}} \equiv \mathfrak{t}_{k_{1}}+\tau \quad\left(\bmod \pi_{0}\right), \ldots, \mathfrak{t}_{k_{c}} \equiv \mathfrak{t}_{k_{1}}+(c-1) \tau \quad\left(\bmod \pi_{0}\right)
$$

- if $\mathfrak{t}_{k}=t_{k}\left(\right.$ resp. $\left.\mathfrak{t}_{k}=t_{k}^{\prime}\right)$, then $z_{k}=x_{j_{k}}$ (resp. $\left.z_{k}=\bar{x}_{l_{k}}\right)$; moreover the subscripts $j_{k}$ (resp. $l_{k}$ ) related to those $x_{j_{k}}$ (resp. $\bar{x}_{l_{k}}$ ) are pairwise distinct. If we are under configuration $\mathbf{C}_{\mathbf{4}}^{\prime}$, the only possible chain of length $\alpha_{0}+1$ is either

$$
\mathfrak{c}_{0}=\left(\left(x_{i}, \tau\right), \ldots,\left(x_{p-1},\left(\alpha_{0}+1\right) \tau\right)\right),
$$


or

$$
\overline{\mathfrak{c}}_{0}=\left(\left(\bar{x}_{j}, \tau\right), \ldots,\left(\bar{x}_{m-1},\left(\alpha_{0}+1\right) \tau\right)\right) .
$$

Notice that, from the definition of $\mathbf{C}_{\mathbf{4}}^{\prime}$ configuration, these two chains, $\mathfrak{c}_{0}$ and $\overline{\mathfrak{c}}_{0}$, cannot coexist. All the other chains have a length less than $\alpha_{0}+1$. To show this fact, let $\left(\left(z_{1}, \mathfrak{t}_{k_{1}}\right), \ldots,\left(z_{c}, \mathfrak{t}_{k_{c}}\right)\right)$ be a chain such that $c=\alpha_{0}+1$. All the elements $\left(z_{1}, \ldots, z_{c}\right)$ belong either to $L_{p-1}$ or to $\bar{L}_{m-1}$ (if not, we could find an equality between an element of $L_{p-1}$ and an element of $\bar{L}_{m-1}$ ). If all the $z_{k}$ 's belong to $L_{p-1}$, then we have $z_{c+1}=z_{1}$; from the definition of $\mathbf{C}_{\mathbf{4}}^{\prime}$ configuration, it follows that $z_{1}=x_{i}$ and $z_{c+1}=x_{p}$. If all the $z_{k}$ 's are in $\bar{L}_{m-1}$, we obtain that $z_{1}=\bar{x}_{j}, z_{c+1}=\bar{x}_{m}$ and $u_{j}=\cdots=u_{m-1}=u_{p-1}$. As all the chain but $\mathfrak{c}_{0}$ have a length less than $\alpha_{0}+1$, we can conclude, as for $\mathbf{C}_{\mathbf{1}}$ configuration, that there exists a function $\mathscr{V}$ defining a vector field $\phi_{0}$ which ensures that $A_{i}=0, \bar{A}_{j}=0$, and $\bar{A}_{m}=0$ while $A_{p}$ can be arbitrarily chosen.

We shall see that a chain of length $\alpha_{0}+1$ is not possible under $\mathbf{C}_{\mathbf{2}}$ or $\mathbf{C}_{\mathbf{3}}^{\prime}$ configuration. Assume that we are not under $\mathbf{C}_{\mathbf{4}}$ configuration and denote by

$$
\mathfrak{c}_{0}=\left(\left(z_{1}, \mathfrak{t}_{1}\right), \ldots,\left(z_{\alpha_{0}+1}, \mathfrak{t}_{\alpha_{0}+1}\right)\right)
$$

a chain with length $\alpha_{0}+1$; notice that, from the definition of a chain, we have

$$
z_{k+1}=\varphi_{\tau}^{u_{p-1}}\left(z_{k}\right)=\varphi_{T}^{u_{p-1}}\left(z_{k}\right) .
$$

There exists $0 \leq \alpha_{1} \leq \alpha_{0}$ such that $\mathfrak{t}_{k_{1}} \equiv-\alpha_{1} \tau\left(\bmod \pi_{0}\right)$ so, from the definition of a chain, we have

$$
\begin{aligned}
\mathfrak{t}_{k_{1}} & \equiv-\alpha_{1} \tau \quad\left(\bmod \pi_{0}\right), \quad \mathfrak{t}_{k_{2}} \equiv-\left(\alpha_{1}-1\right) \tau \quad\left(\bmod \pi_{0}\right), \ldots, \\
\mathfrak{t}_{k_{\alpha_{0}+1}} & \equiv-\left(\alpha_{1}-\alpha_{0}\right) \tau \quad\left(\bmod \pi_{0}\right)
\end{aligned}
$$

therefore $\mathfrak{t}_{k_{\alpha_{1}+1}} \equiv 0\left(\bmod \pi_{0}\right)$ and $z_{\alpha_{1}+1}=x_{p-1}$. If $\alpha_{1}<\alpha_{0}$, this equality implies $z_{\alpha_{1}+2}=x_{p}$; if $\alpha_{1}=\alpha_{0}$, we have $z_{1}=\varphi_{-\alpha_{0} \tau}^{u_{p-1}}\left(x_{p-1}\right)=\varphi_{\tau}^{u_{p-1}}\left(x_{p-1}\right)=x_{p}$. Thus, in chain $\mathfrak{c}_{0}$, there exists an element $z_{i}$ equal to $x_{p}$, this implies that we cannot be under $\mathbf{C}_{\mathbf{2}}$ configuration because, in this case we can neither have $z_{i}=x_{j_{k}}$ because $j_{k}<p$ nor $z_{i}=x_{p}=\bar{x}_{m}=\bar{x}_{l_{k}}$ because $l_{k}<m$. Thus we are under $\mathbf{C}_{\mathbf{3}}^{\prime}$ configuration, reordering the elements of the chain, we can assume that

$$
\mathfrak{t}_{k_{1}} \equiv-\alpha_{0} \tau \quad\left(\bmod \pi_{0}\right), \mathfrak{t}_{k_{2}} \equiv-\left(\alpha_{0}-1\right) \tau \quad\left(\bmod \pi_{0}\right), \ldots, \mathfrak{t}_{k_{\alpha_{0}+1}} \equiv 0 \quad\left(\bmod \pi_{0}\right) .
$$

We have $z_{1}=\varphi_{-\alpha_{0} \tau}^{u_{p-1}}\left(x_{p-1}\right)=\varphi_{\tau}^{u_{p-1}}\left(x_{p-1}\right)=x_{p}$, so, as all the subscripts $j_{k}$ are less than $p$, we have $z_{1}=\bar{x}_{m} \in \bar{L}_{2 n}$. Let $r$ be the greatest subscript such that $z_{1}, \ldots, z_{r} \in$ $\bar{L}_{2 n}$; from (35), we have $z_{r}=\bar{x}_{m+r-1}$ and $z_{r+1}=x_{j_{k}}$ for some subscript $j_{k}<p$, so $x_{j_{k}}=\bar{x}_{m+r}$; from the definition of configuration $\mathbf{C}_{\mathbf{3}}^{\prime}$ this implies that we cannot have $m+r<j$, so $m+r=j$ and $z_{r+1}=x_{i}$. Let $s$ be the greatest subscript greatest than or equal to $r+1$ such that $z_{r+1}, \ldots, z_{s} \in L_{2 n}$. We have $z_{s}=x_{s+i-r-1}$ and we claim that $z_{s}$ is the last element of $\mathfrak{c}_{0}$ because if there exist $z_{s+1} \in \bar{L}_{2 n}$, then we have $z_{s+1}=\varphi_{\tau}^{u_{p-1}}\left(z_{s}\right)=x_{s+i-r}$; from the definition of configuration $\mathbf{C}_{\mathbf{3}}^{\prime}$ this implies that $s+i-r=p$ and $z_{s+1}=\bar{x}_{m}$ which contradicts the definition of a chain. The element $z_{s}$ being the last element of the chain, we have $z_{s}=\varphi_{\alpha_{0} \tau}^{u_{p-1}}\left(z_{1}\right)=\varphi_{-\tau}^{u_{p-1}}\left(\bar{x}_{m}\right)=x_{p-1}$, therefore chain $\mathfrak{c}_{0}$ can be written

$\mathfrak{c}_{0}=\left(\left(\bar{x}_{m},-\alpha_{0} \tau\right), \ldots,\left(\bar{x}_{j-1},-\left(\alpha_{0}-j+m+1\right) \tau\right),\left(x_{i},-\left(\alpha_{0}-j+m\right) \tau\right), \ldots,\left(x_{p-1}, 0\right)\right)$

from which we deduce that $u_{i}=\cdots=u_{p-1}=u_{m}=\cdots=u_{j-1}$ which contradicts the definition of $\mathbf{C}_{\mathbf{3}}^{\prime}$. 
[1] Ralph Abraham and Joel Robbin. Transversal Mappings and Flows. W. A. Benjamin, New York, 1967.

[2] Dirk Aeyels. Generic observability of differentiable systems. SIAM Journal on Control and Optimization, 19(5):595-603, 1981.

[3] Sabeur Ammar, Hind Feki, and Jean-Claude Vivalda. Observability under sampling for bilinear system. International Journal of Control, 87(2):312-319, 2014.

[4] Sabeur Ammar, Mohamed Mabrouk, and Jean-Claude Vivalda. On the genericity of the differential observability of controlled discrete-time systems. SIAM Journal on Control and Optimization, 46(6):2182-2198, 2007.

[5] Sabeur Ammar, Majid Massaoud, and Jean-Claude Vivalda. Observability under sampling for nonlinear systems. Asian Journal of Control, 18(4):1269-1278, 2016.

[6] Sabeur Ammar and Jean-Claude Vivalda. On the preservation of observability under sampling. Systems \& Control Letters, 52(1):7-15, 2004.

[7] Sabeur Ammar and Jean-Claude Vivalda. On the genericity of the observability of controlled discrete-time systems. ESAIM: Control, Optimisation and Calculus of Variations, 11(02):161-179, 2005.

[8] Éric Busvelle and Jean-Paul Gauthier. On determining unknown functions in differential systems, with an application to biological reactors. ESAIM: Control, Optimisation and Calculus of Variations, 9:509-551, 2003.

[9] Jean-Paul Gauthier, Hassan Hammouri, and Ivan Kupka. Observers for nonlinear systems. In Proceedings of the 30th IEEE Conference on Decision and Control, volume 2, pages 1483-1489, dec 1991.

[10] Jean-Paul Gauthier and Ivan Kupka. Observability for systems with more outputs than inputs and asymptotic observers. Mathematische Zeitschrift, 223:47-78, 1996.

[11] Jean-Paul Gauthier and Ivan Kupka. Deterministic Observation Theory and Applications. Cambridge University Press, Cambridge, 2001.

[12] M. Golubitsky and V. Guillemin. Stable Mappings and Their Singularities. Graduate Texts in Mathematics. Springer Verlag, 1986.

[13] Jeremy P. Huke and David S. Broomhead. Embedding theorems for non-uniformly sampled dynamical systems. Nonlinearity, 20(9):2205, 2007.

[14] Gerhard Kreisselmeier. On sampling without loss of observability/controllability. Automatic Control, IEEE Transactions on, 44(5):1021-1025, may 1999.

[15] Ivan Kupka. Contribution à la théorie des champs génériques. Contributions to Differential Equations, 2:457-484, 1963.

[16] Ivan Kupka. Addendum et corrections au mémoire: "Contributions à la théorie des champs génériques". Contributions to Differential Equations, 3:411-420, 1964.

[17] Stephen Smale. Stable manifolds for differential equations and diffeomorphisms. Ann. Scuola Norm. Sup. Pisa (3), 17:97-116, 1963.

[18] Eduardo D. Sontag. Mathematical Control Theory: Deterministic Finite Dimensional Systems. Texts in Applied Mathematics. Springer, New York, 1998.

[19] Jorge Sotomayor. Generic one-parameter families of vector fields on two-dimensional manifolds. Inst. Hautes Études Sci. Publ. Math., 43(1):5-46, 1974.

[20] Jean-Claude Vivalda. On the genericity of the observability of uncontrolled discrete nonlinear systems. SIAM Journal on Control and Optimization, 42(4):1509-1522, 2003. 